\title{
An Exponentially Convergent Nonpolynomial Finite Element Method for Time-Harmonic Scattering from Polygons
}

Article

Published Version

Banett, A.H. and Betcke, T. (2010) An Exponentially Convergent Nonpolynomial Finite Element Method for TimeHarmonic Scattering from Polygons. Siam Journal on Scientific Computing, 32 (3). pp. 1417-1441. ISSN 1064-8275 doi: https://doi.org/10.1137/090768667 Available at https://centaur.reading.ac.uk/17191/

It is advisable to refer to the publisher's version if you intend to cite from the work. See Guidance on citing.

Published version at: http://dx.doi.org/10.1137/090768667

To link to this article DOI: http://dx.doi.org/10.1137/090768667

Publisher: Society for Industrial and Applied Mathematics

All outputs in CentAUR are protected by Intellectual Property Rights law, including copyright law. Copyright and IPR is retained by the creators or other copyright holders. Terms and conditions for use of this material are defined in the End User Agreement.

www.reading.ac.uk/centaur 
Central Archive at the University of Reading

Reading's research outputs online 


\title{
AN EXPONENTIALLY CONVERGENT NONPOLYNOMIAL FINITE ELEMENT METHOD FOR TIME-HARMONIC SCATTERING FROM POLYGONS*
}

\author{
A. H. BARNETT ${ }^{\dagger}$ AND T. BETCKE
}

\begin{abstract}
In recent years nonpolynomial finite element methods have received increasing attention for the efficient solution of wave problems. As with their close cousin the method of particular solutions, high efficiency comes from using solutions to the Helmholtz equation as basis functions. We present and analyze such a method for the scattering of two-dimensional scalar waves from a polygonal domain that achieves exponential convergence purely by increasing the number of basis functions in each element. Key ingredients are the use of basis functions that capture the singularities at corners and the representation of the scattered field towards infinity by a combination of fundamental solutions. The solution is obtained by minimizing a least-squares functional, which we discretize in such a way that a matrix least-squares problem is obtained. We give computable exponential bounds on the rate of convergence of the least-squares functional that are in very good agreement with the observed numerical convergence. Challenging numerical examples, including a nonconvex polygon with several corner singularities, and a cavity domain, are solved to around 10 digits of accuracy with a few seconds of CPU time. The examples are implemented concisely with MPSpack, a MATLAB toolbox for wave computations with nonpolynomial basis functions, developed by the authors. A code example is included.
\end{abstract}

Key words. scattering, polygons, Helmholtz equation, fundamental solutions

AMS subject classifications. 65N30, 35J05

DOI. $10.1137 / 090768667$

1. Introduction. Nonpolynomial finite element methods for wave problems such as the PUFEM [3], the least-squares finite element method [24], or the ultraweak variational formulation [8] have recently received much attention in the mathematics and engineering communities. The idea of these methods is to use Trefftz-type basis functions, i.e., exact solutions to the PDE inside each element, then solve for the coefficients which best satisfy the interelement matching and/or boundary conditions. This better captures solution behavior than standard polynomial bases, and it is closely related to the method of particular solutions or collocation methods [23]. Typical choices of basis functions are plane waves [26], Fourier-Bessel expansions [14, 27], and fundamental solutions of the Helmholtz equation [6, 16, 4].

The main advantage of nonpolynomial finite element methods over standard Galerkin methods is that they allow one to choose element sizes which are many wavelengths in diameter, while standard Galerkin methods with linear elements typically need of order 10 elements per wavelength to resolve a wave to any reasonable accuracy. Thus at high frequency the total number of degrees of freedom is much reduced, and due to the high-order (often exponential) convergence of nonpolynomial finite element methods, this advantage is even more pronounced if high accuracy is required.

*Received by the editors August 19, 2009; accepted for publication (in revised form) February 23, 2010; published electronically May 21, 2010.

http://www.siam.org/journals/sisc/32-3/76866.html

${ }^{\dagger}$ Department of Mathematics, Dartmouth College, Hanover, NH 03755 (ahb@math.dartmouth. edu). The work of this author was supported by National Science Foundation grant DMS-0811005.

$\ddagger$ Department of Mathematics, University of Reading, Reading, Berkshire RG6 6AH, UK (t.betcke@reading.ac.uk). The work of this author was supported by Engineering and Physical Sciences Research Council grant EP/F067798/1. 
Great progress has also been made in recent years in the development of wavenumber independent boundary integral equation methods for the solution of scattering problems (see, for example, $[7,18,10,13])$. An excellent overview of these new methods is given in [9].

In this paper we analyze a nonpolynomial finite element method for time-harmonic scattering from a sound-soft polygonally shaped bounded obstacle $\Omega \subset \mathbb{R}^{2}$. That is, we are looking for the solution $u$ of the problem

$$
\begin{aligned}
\Delta u+k^{2} u & =0 \quad \text { in } \mathbb{R}^{2} \backslash \bar{\Omega}, \\
u & =0 \quad \text { on } \partial \Omega, \\
\frac{\partial u_{s}}{\partial r}-i k u_{s} & =o\left(r^{-1 / 2}\right),
\end{aligned}
$$

where $u=u_{i n c}+u_{s}$ is the total field in which $u_{i n c}$ is the incident wave and $u_{s}$ the scattered field, and where $r$ is the radial coordinate. The wavenumber is $k=$ $2 \pi / \lambda$, where $\lambda$ is the wavelength. The Sommerfeld radiation condition (1.3) is to be understood to hold uniformly in all directions.

The main idea of the presented method is to use fractional order Fourier-Bessel functions at the corners of the polygon to match the asymptotic behavior of $u$ there, and to represent the scattered field $u_{s}$ towards infinity by fundamental solutions (i.e., effective sources), which automatically satisfy (1.3). The matching conditions between different elements are implemented using a least-squares finite element formulation. A related approach was proposed without analysis by Stojek in [27]. The main differences in our formulation are the choice of basis functions towards infinity and the setup of the discretized problem.

Least-squares finite element methods were also analyzed by Monk and Wang in [24]. Their analysis focused on Helmholtz problems on smooth domains with local absorbing boundary conditions. For such problems they also proved interior error estimates based on the least-squares functional.

In this paper we choose each element as large as possible in such a way that each of the finite subdomains contains one corner of the polygon. The accuracy is then improved by increasing the number of basis functions in each subdomain. Hence, $h$-refinement is not necessary. This is similar to the domain decomposition method of Descloux and Tolley for the Laplace eigenvalue problem on polygonal domains [12].

We give exponential convergence bounds with a rate that can be numerically computed using techniques from conformal maps (see also [5]). The asymptotic convergence rate is wavenumber-independent. However, we observe that the minimum number of basis functions $N$ required to enter into the regime of exponential convergence depends linearly on $k$, leading to a complexity of $O\left(k^{3}\right)$ for the fully discrete scheme due to the need for solving dense linear systems. But due to the fast exponential convergence, and the relatively small preprocessing effort, the overall computation time for this method is very attractive and is competitive for low to medium frequencies with the wavenumber-independent approach recently introduced by ChandlerWilde and Langdon [10]. Furthermore, the method presented here works on convex and nonconvex polygons, while the approach in [10] is restricted to convex polygons. We will demonstrate that high accuracy (up to 10 digits) is easily achievable.

Another advantage of the proposed approach compared to boundary element (or boundary integral) methods is that no quadrature of singular integrals close to the corners is necessary. Furthermore, the proposed method can be easily adapted to the sound-hard scattering case by a small change in the basis functions close to the 

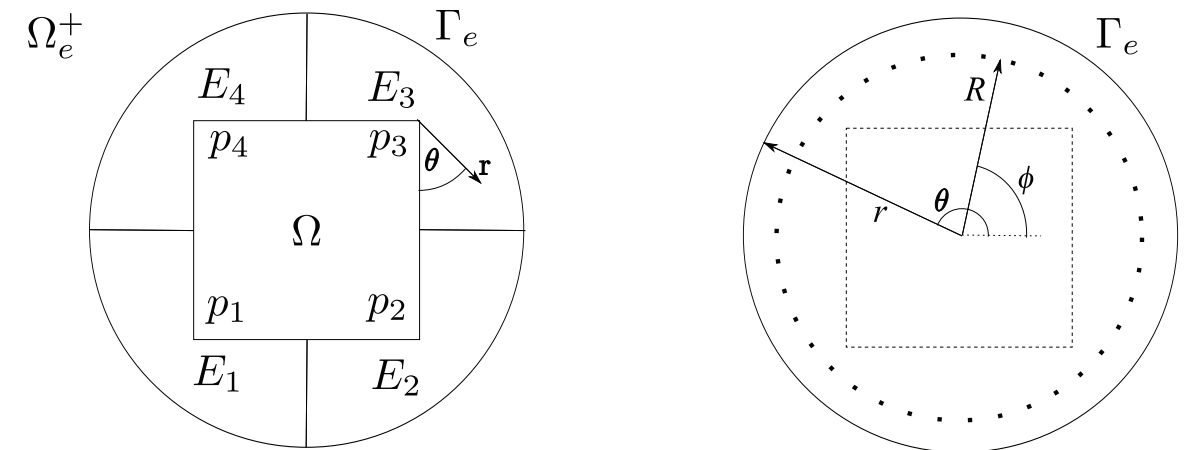

FIG. 2.1. Geometry of subdomains, illustrated for the square scatterer. For the exterior angles $\pi / \alpha_{1}, \ldots, \pi / \alpha_{4}$ at the corners $p_{1}, \ldots, p_{4}$, we have in this example that $\alpha_{1}=\cdots=\alpha_{4}=2 / 3$. Left plot: The local coordinate systems of the Fourier-Bessel basis functions are oriented along the boundary lines adjacent to the corners of the polygon. Right plot: Fundamental solutions basis coordinate system, showing the charge points $\boldsymbol{y}_{j}$ lying on a circle.

corners. In contrast, combined integral formulations for sound-hard scattering involve the evaluation of hypersingular integral operators.

The paper is organized as follows. In section 2 we present the formulation of the method. Section 3 describes how we discretize this into a linear system. The approximation of the scattered field $u_{s}$ towards infinity is done using fundamental solutions approximations, which are presented in more detail in section 4 . In particular, a combined monopole-plus-dipole formulation is given that avoids problems with interior resonances. The convergence analysis in section 5 depends on approximation theoretic results for Bessel functions and an analysis of the convergence of the fundamental solutions approximations. A detailed analysis of the exponential convergence of fractional order Fourier-Bessel functions in cornered domains based on techniques from complex analysis was given by Betcke in [5]. Here, we state only the main convergence result of that paper adapted to our situation. Numerical examples are given in section 6. All computations are implemented quite simply in MPSpack, a MATLAB toolbox for wave computations developed by the authors; a short example code to compute scattering from the square and plot Figure 6.3 is given in Appendix A. Finally, since maintaining reasonably small coefficients is important for high accuracy, the numerical stability of the method is discussed in section 7 . We conclude in section 8 .

2. Formulation of the method. Let $\Omega$ be a polygonal domain with boundary $\Gamma$ having $q$ corners at the points $p_{1}, \ldots, p_{q} \in \mathbb{R}^{2}$ and associated exterior angles $0<$ $\pi / \alpha_{j}<2 \pi, j=1, \ldots, q$. We define the artificial exterior boundary $\Gamma_{e}:=\partial \Omega_{e}$ to be an analytic Jordan curve whose simply connected interior $\Omega_{e}$ completely contains $\bar{\Omega}$, i.e., $\operatorname{dist}\left(\Gamma, \Gamma_{e}\right)>0$. We will also frequently need the exterior domain $\Omega_{e}^{+}=\mathbb{R}^{2} \backslash \overline{\Omega_{e}}$. Also, denote by $E:=\Omega_{e} \backslash \bar{\Omega}$ the part of $\Omega_{e}$ exterior to $\Omega$. The geometry and notation are shown in Figure 2.1.

We subdivide $E$ into $q$ simply connected subdomains $E_{i}, i=1, \ldots, q$, with boundaries $\Gamma_{i}=\partial E_{i}$ in such a way that the following conditions are satisfied:

- $E_{i} \cap E_{j}=\emptyset$ for all $i \neq j$,

- $\bigcup_{i} \overline{E_{i}}=\bar{E}$,

- $\Gamma_{i} \cap \partial \Omega$ consists of two straight lines which meet at the corner $p_{i}$ and whose continuation towards infinity does not intersect $E_{i}{ }^{1}$

\footnotetext{
${ }^{1}$ The continuation condition is a technical condition needed for the convergence estimates on the finite subdomains (see [5, Fig. 6] for an example violating this condition).
} 
Remark 2.1. The requirement that every subdomain $E_{i}$ contain a corner of $\Omega$ is not strictly necessary and is used only to simplify the description. An extension to the case of "free" elements that have no intersection with $\Omega$ is straightforward. This is used in the cavity example in section 6.3.

In each subdomain $E_{i}$ we approximate the total field $u$ by a linear combination of Fourier-Bessel functions of the form

$$
u(r, \theta) \approx \sum_{j=1}^{N_{i}} c_{j}^{(i)} J_{j \alpha_{i}}(k r) \sin j \alpha_{i} \theta
$$

The local polar coordinate systems of the Fourier-Bessel functions are shown in Figure 2.1. This guarantees that the basis functions automatically satisfy the zero boundary conditions on $\Gamma$. We denote by $V_{i}$ the finite dimensional linear space spanned by all Fourier-Bessel functions of the form (2.1) in the subdomain $E_{i}$.

The approximation by Fourier-Bessel functions is motivated by the corner behavior of Helmholtz solutions. One can show that in a wedge with interior angle $\pi / \alpha$, any Helmholtz solution $u$ that satisfies zero Dirichlet boundary conditions on the sides of the wedge has close to the corner the absolutely convergent expansion

$$
u(r, \theta)=\sum_{j=1}^{\infty} c_{j} J_{\alpha j}(k r) \sin \alpha j \theta
$$

for certain coefficients $c_{j} \in \mathbb{C}$ [12]. If $\alpha \notin \mathbb{N}$, then any nontrivial $u$ has a singularity at the corner of the wedge. Otherwise, if $\alpha \in \mathbb{N}$, then $u$ can be analytically continued across the corner of the wedge [23].

In $\Omega_{e}^{+}$we approximate as follows the scattered field $u_{s}$ by a linear combination of fundamental solutions whose origins lie in $\Omega_{e}$. Let $\Gamma_{F} \subset E$ be a closed analytic Jordan curve, and choose points $\mathbf{y}_{j} \in \Gamma_{F}$. Then our approximation, which satisfies the Helmholtz equation in $\Omega_{e}^{+}$, is

$$
u_{s}(\mathbf{x}) \approx \sum_{j=1}^{N_{e}} c_{j}^{(e)}\left(\frac{i}{4} \frac{\partial}{\partial \nu\left(\mathbf{y}_{j}\right)} H_{0}^{(1)}\left(k\left|\mathbf{x}-\mathbf{y}_{j}\right|\right)-\frac{\eta}{4} H_{0}^{(1)}\left(k\left|\mathbf{x}-\mathbf{y}_{j}\right|\right)\right), \quad \mathbf{x} \in \Omega_{e}^{+},
$$

where $\nu\left(\mathbf{y}_{j}\right)$ is the outward-facing unit normal direction of the curve $\Gamma_{F}$ at the point $\mathbf{y}_{j}, H_{0}^{(1)}$ is the outgoing Hankel function of zeroth order, and $\eta \neq 0$ is a real parameter. Typically, by a scaling argument we choose $\eta=k$. Formulation (2.2) can be interpreted as a discrete version of an integral equation of the first kind for approximating $u$ (see section 4). The finite dimensional space of all linear combinations of the fundamental solutions in $\Omega_{e}^{+}$of form (2.2) is denoted by $V_{e}$.

Remark 2.2. One could alternatively approximate the scattered field $u_{s}$ by a multipole expansion of the form

$$
u_{s}(r, \theta) \approx \sum_{m=-N_{e}}^{N_{e}} \alpha_{m} H_{m}^{(1)}(k r) e^{i m \theta} .
$$

This was proposed by Stojek in [27]. The disadvantage of this expansion is that when $\Gamma_{e}$ is anything other than a circle about the origin, severe numerical stability problems arise at large wavenumber due to the huge dynamic range of Hankel functions at large $m$. Fundamental solutions do not suffer from this problem, and hence allow more flexibility (e.g., see section 6.2). 
Combining the above basis sets, the trial space $V$ of the finite element method is the space of functions $v$ such that $v_{i}:=\left.v\right|_{E_{i}} \in V_{i}$ for all $i$, and $v_{e}:=\left.v\right|_{\Omega_{e}^{+}} \in V_{e}$. It is useful to express the number of basis functions in each subdomain as a multiplier of a common factor $N$. Let $N_{i}=n_{i} N, i=1, \ldots, q$, and $N_{e}=n_{e} N$. The total dimension of the trial space is then

$$
N_{V}=\left[n_{e}+\sum_{i=1}^{q} n_{i}\right] N=: n_{V} N .
$$

We now explain the least-squares finite element formulation as proposed by Stojek in [27] and Monk and Wang in [24]. Let $\Gamma_{i j}$ be the internal boundary between two elements $E_{i}$ and $E_{j}$. Let $\nu(\mathbf{x})$ be a unit normal to the curve $\Gamma_{i j}, i<j$, defined for almost every $\mathrm{x} \in \Gamma_{i j}$, pointing away from domain $E_{i}$. The jump of a function $u$ defined on $E_{i}$ and $E_{j}$ is

$$
[u](\mathbf{x}):=\lim _{\epsilon \rightarrow 0} u(\mathbf{x}+\epsilon \nu(\mathbf{x}))-u(\mathbf{x}-\epsilon \nu(\mathbf{x})), \quad \mathbf{x} \in \Gamma_{i j} .
$$

Denoting by $\partial_{\nu}:=\nabla \cdot \nu$ the normal derivative in the sense explained above, and by $\left[\partial_{\nu} u\right]$ its jump, define the interelement matching error functional

$$
\begin{aligned}
J(v):= & \sum_{i<j} \int_{\Gamma_{i j}}\left|\left[\partial_{\nu} v\right]\right|^{2} d s+k^{2}|[v]|^{2} d s \\
& +\sum_{i=1}^{q} \int_{\Gamma_{i} \cap \Gamma_{e}}\left|\left[\partial_{\nu}\left(\hat{u}_{i n c}+v\right)\right]\right|^{2}+k^{2}\left|\left[\hat{u}_{i n c}+v\right]\right|^{2} d s,
\end{aligned}
$$

where

$$
\hat{u}_{i n c}(\mathbf{x}):= \begin{cases}u_{i n c}(\mathbf{x}), & \mathbf{x} \in \Omega_{e}^{+} \\ 0, & \mathbf{x} \in \Omega_{e}\end{cases}
$$

Here we need the restriction $\hat{u}_{i n c}$ of the incoming wave $u_{\text {inc }}$ to $\Omega_{e}^{+}$since inside $\Omega_{e}$ we directly approximate the full field $u$, while in $\Omega_{e}^{+}$we approximate the scattered field $u_{s}$.

The least-squares finite element approximation $v_{L S}$ is now defined as

$$
v_{L S}=\arg \min _{v \in V} J(v) .
$$

3. Implementation. In this section we give details of the numerical implementation of the least-squares formulation (2.6). Denote by $c=\left[c^{(1)}, \ldots, c^{(q)}, c^{(e)}\right]^{T} \in$ $\mathbb{C}^{n_{V} N}$ the coefficient vector associated with all basis functions in the elements $E_{i}$, $i=1, \ldots, q$, and $\Omega_{e}^{+}$. Since the basis functions in each element are linearly independent, every $v \in V$ has a unique representation in terms of a vector $c \in \mathbb{C}^{n_{V} N}$. We can therefore reformulate (2.6) equivalently as

$$
c_{L S}=\arg \min _{c \in \mathbb{C}^{N_{V}}} J(c) .
$$

For notational convenience we use the same name for the least-squares functional $J(v)$ defined in (2.4) and its parameterized version in terms of a coefficient vector $c$.

We now derive a matrix representation of $J(c)$. Consider first two elements $E_{i}$ and $E_{j}$, within which basis functions are $g_{1}^{(i)}, \ldots, g_{N_{i}}^{(i)}$ and $g_{1}^{(j)}, \ldots, g_{N_{j}}^{(j)}$, respectively. 
We assume that these functions and their derivatives are also defined on $\Gamma_{i j}$. Denote by $\xi_{\tau}, \tau=1, \ldots, m_{i j}$, quadrature points on $\Gamma_{i j}$ with corresponding weights $\omega_{\tau}>0$ appropriate for integration with respect to arc length (e.g., in section 6 we use the Clenshaw-Curtis rule). Then from $\Gamma_{i j}$ the contribution to $J(c)$ is

$$
\begin{aligned}
& \int_{\Gamma_{i j}} k^{2}\left|\sum_{p=1}^{N_{i}} c_{p}^{(i)} g_{p}^{(i)}(s)-\sum_{p=1}^{N_{j}} c_{p}^{(j)} g_{p}^{(j)}(s)\right|^{2}+\left|\sum_{p=1}^{N_{i}} c_{p}^{(i)} \partial_{\nu} g_{p}^{(i)}(s)-\sum_{p=1}^{N_{j}} c_{p}^{(j)} \partial_{\nu} g_{p}^{(j)}(s)\right|^{2} d s \\
& \approx\left\|\left[\begin{array}{ll}
W_{i j} & \\
& W_{i j}
\end{array}\right]\left[\begin{array}{cc}
k A_{i} & -k A_{j} \\
\partial_{\nu} A_{i} & -\partial_{\nu} A_{j}
\end{array}\right]\left[\begin{array}{c}
c^{(i)} \\
c^{(j)}
\end{array}\right]\right\|_{2}^{2},
\end{aligned}
$$

where for each $i=1, \ldots, q, A_{i}$ is the matrix with elements $\left(A_{i}\right)_{\tau p}=g_{p}^{(i)}\left(\xi_{\tau}\right)$, and $\left(\partial_{\nu} A_{i}\right)_{\tau p}=\partial_{\nu} g_{p}^{(i)}\left(\xi_{\tau}\right)$ is the matrix of normal derivatives. Quadrature weights now reside in the diagonal matrix $W_{i j}$ with elements $\left(W_{i j}\right)_{\tau \tau}=\omega_{\tau}^{1 / 2}, \tau=1, \ldots, m_{i j}$. On each outer boundary the contribution to $J(c)$ is

$$
\begin{aligned}
& \int_{\Gamma_{i} \cap \Gamma_{e}} k^{2}\left|\sum_{p=1}^{N_{i}} c_{p}^{(i)} g_{p}^{(i)}(s)-\sum_{p=1}^{N_{e}} c_{p}^{(e)} g_{p}(s)-u_{i n c}(s)\right| \\
& +|| \sum_{p=1}^{N_{i}} c_{p}^{(i)} \partial_{\nu} g_{p}^{(i)}(s)-\sum_{p=1}^{N_{e}} c_{p}^{(e)} \partial_{\nu} g_{p}(s)-\left.\partial_{\nu} u_{i n c}(s)\right|^{2} d s \\
& \approx\left\|\left[\begin{array}{ll}
W_{i e} & \\
& W_{i e}
\end{array}\right]\left(\left[\begin{array}{cc}
k A_{i} & -k A_{e} \\
\partial_{\nu} A_{i} & -\partial_{\nu} A_{e}
\end{array}\right]\left[\begin{array}{c}
c^{(i)} \\
c^{(j)}
\end{array}\right]-\left[\begin{array}{c}
b_{i} \\
\tilde{b}_{i}
\end{array}\right]\right)\right\|_{2}^{2},
\end{aligned}
$$

where $g_{\ell}$ is the $\ell$ th fundamental solutions basis function with source at $\mathbf{y}_{\ell}$, and, analogous to the above, $\left(A_{e}\right)_{\tau p}=g_{p}\left(\xi_{\tau}\right),\left(\partial_{\nu} A_{e}\right)_{\tau p}=\partial_{\nu} g_{p}\left(\xi_{\tau}\right)$, and quadrature points now live on $\Gamma_{i} \cap \Gamma_{e}$. The inhomogeneous vector is filled with the known values $\left(b_{i}\right)_{\tau}=$ $u_{i n c}\left(\xi_{\tau}\right)$ and $\left(\tilde{b}_{i}\right)_{\tau}=\partial_{\nu} u_{i n c}\left(\xi_{\tau}\right)$ for $i=1, \ldots, m_{i e}$.

Note that, by construction, all of the above quadratures involve smooth (in fact analytic) functions on the respective segments; no quadrature near the corner singularities is needed. However, the integrand is oscillatory on the wavelength scale, and this will inform the choice of the number of quadrature nodes.

Stacking up all contributions from the different interfaces, we obtain

$$
J(c) \approx\|W(A c-b)\|_{2}^{2} .
$$

The matrix $A$ is built up from the subblocks $A_{i}$ and $\partial_{\nu} A_{i}$ as described above, and the right-hand side vector $b$ is zero apart from the contributions of the incident wave at the quadrature points on $\Gamma_{e}$. In $[27,24,17]$ the authors discretize the variational derivative of $J(v)$ to obtain a linear system of equations that they solve. This approach is equivalent to solving the normal equation

$$
A^{H} W^{2} A c=A^{H} W^{2} b .
$$

However, as long as the problem size is not too large we can, and do, equivalently solve directly the least squares problem

$$
\min _{c \in \mathbb{C}^{N_{V}}}\|W(A c-b)\|_{2}
$$

using a dense least-squares solver, such as the backslash (mldivide) in MATLAB. This is numerically more stable than solving (3.1) if $W A$ is ill-conditioned. 
4. The method of fundamental solutions for scattering problems. In this section we give a brief introduction to the method of fundamental solutions (MFS) for Helmholtz problems in exterior domains and motivate the combined MFS formulation (2.2). Overviews of the MFS, its theory, and applications are given in [6, 15]. The results of this section form the basis for the convergence analysis in section 5.1.

Let $D$ be a simply connected domain with analytic boundary $\partial D$. Let $u_{s}$ be the unique solution [11] to the Helmholtz equation (1.1) in $\mathbb{R}^{2} \backslash \bar{D}$ and the Sommerfeld condition (1.3) with boundary value data $u_{s}=f$ on $\partial D$. The general idea of the MFS is to choose charge points in $D$, with strengths (coefficients), whose combined field can well approximate $u_{s}$ in $\mathbb{R}^{2} \backslash \bar{D}$ as the number of charges grows. We focus on the special case where these charges lie on a closed curve $\Gamma_{F} \subset D$ some distance inside $D$. Then the MFS may be viewed as a quadrature approximation to a single-layer potential of the form

$$
u_{s}(\mathbf{x}) \approx \int_{\Gamma_{F}} \frac{i}{4} H_{0}^{(1)}(k|\mathbf{x}-\mathbf{y}|) g(\mathbf{y}) d s_{\mathbf{y}} .
$$

However, the MFS is more general than merely a discretization of a standard boundary integral representation: depending on the smoothness of $u_{s}$ (or singularities of its continuation into $D$ ), there might not exist any layer density $g$ that can represent $u_{s}$ exactly. Nevertheless there still may exist a sequence of densities in suitable spaces whose field converges to $u_{s}$. The discrete version of this statement is that, with the MFS, there exists a sequence of charge points and strengths whose field converges to $u_{s}$. If $\Gamma_{F}$ is suitably chosen, one can show that there are such sequences that give rapid exponential convergence in certain norms (see [4] and our section 5.1).

Unlike boundary integral methods which enable a second kind formulation, (4.1) is necessarily first kind. However, it has the advantages of easily provable convergence rate, of simplicity of evaluation arbitrarily close to $\partial D$, and of no singular quadrature schemes being required. Disadvantages of the MFS are that the right choice of $\Gamma_{F}$ and the quadrature points $\mathbf{y}_{j}$ on $\Gamma_{F}$ can be difficult for complicated domains, and that the MFS typically leads to ill-conditioned linear systems. In our case the exterior boundary $\Gamma_{e}$ is typically a very simple shape (circles or simple analytic curves), making it easy to automate the choice of $\Gamma_{F}$, and the effects of the ill-conditioning can be controlled in such a way that computing $u$ to many digits of accuracy is still possible (see section 7).

We now focus on the case that $D$ is a disk with radius $r$ and $\Gamma_{F}$ is a circle with radius $R<r$ (see Figure 2.1, which shows this geometry for the case $D=\Omega_{e}$ that we will use in section 5.1). We identify the plane $\mathbb{R}^{2}$ with $\mathbb{C}$. Define the single-layer operator $S: L^{2}[0,2 \pi] \rightarrow L^{2}[0,2 \pi]$ by

$$
(S g)(\theta)=\int_{0}^{2 \pi} \frac{i}{4} H_{0}^{(1)}\left(k\left|r e^{i \theta}-R e^{i \phi}\right|\right) g(\phi) d \phi .
$$

The task is to find a density $g$ such that the boundary data is matched, that is, $S g \approx f$. For the interior Helmholtz problem on the disk, this was analyzed in [4]. Here, we focus on the eigenvalue expansion of $S$ in order to reveal some stability properties for exterior problems and to lay the groundwork for section 5.1.

Using Graf's addition formula [1, eq. 9.1.79] we obtain

$$
\frac{i}{4} H_{0}^{(1)}\left(k\left|r e^{i \theta}-R e^{i \phi}\right|\right)=\sum_{m \in \mathbb{Z}} \frac{i}{4} H_{m}^{(1)}(k r) e^{-i m \phi} J_{m}(k R) e^{i m \theta} .
$$


Defining the Fourier coefficient $\hat{g}(m)$ of a function $g \in L^{2}[0,2 \pi]$ by $\hat{g}(m)=\frac{1}{2 \pi} \int_{0}^{2 \pi}$ $g(\phi) e^{-i m \phi} d \phi$, it follows that

$$
(S g)(\theta)=\sum_{m \in \mathbb{Z}} \hat{s}(m) \hat{g}(m) e^{i m \theta},
$$

where the eigenfunctions of $S$ are the complex exponentials, and the eigenvalues of $S$ are

$$
\hat{s}(m)=\frac{i \pi}{2} H_{m}^{(1)}(k r) J_{m}(k R) .
$$

If $k R$ hits a zero of the Bessel function of order $m$, then $\hat{s}(m)=0$. This occurs if and only if $k^{2}$ is a Dirichlet eigenvalue of the disk of radius $R$. But the exterior Helmholtz problem has a unique solution for all $k>0$, and therefore we need a formulation that is not affected by interior resonances. We achieve this by a combined formulation analogous to those for boundary integral equations [11].

We define the modified operator $S_{\eta}: L^{2}[0,2 \pi] \rightarrow L^{2}[0,2 \pi]$ by

$$
\left(S_{\eta} g\right)(\theta)=\frac{i}{4} \int_{0}^{2 \pi}\left(\frac{\partial}{\partial R} H_{0}^{(1)}\left(k\left|r e^{i \theta}-R e^{i \phi}\right|\right)+i \eta H_{0}^{(1)}\left(k\left|r e^{i \theta}-R e^{i \phi}\right|\right)\right) g(\phi) d \phi .
$$

This combines single- and double-layer densities. Its eigenvalues $\hat{s}_{\eta}(m)$ are easily computed as

$$
\begin{aligned}
\hat{s}_{\eta}(m) & =\frac{\pi}{2} H_{m}^{(1)}(k r)\left[i \frac{\partial}{\partial R} J_{m}(k R)-\eta J_{m}(k R)\right] \\
& =\frac{\pi}{2} H_{m}^{(1)}(k r)\left[\frac{i k}{2}\left(J_{m-1}(k R)-J_{m+1}(k R)\right)-\eta J_{m}(k R)\right] .
\end{aligned}
$$

Lemma 4.1. Let $\eta \in \mathbb{R} \backslash\{0\}$. Then $\hat{s}_{\eta}(m) \neq 0$ for all $m \in \mathbb{Z}$.

Proof. For Hankel functions of the first kind, it holds that $\left|H_{m}^{(1)}(z)\right| \neq 0$ for all $z \in \mathbb{C}\left[25\right.$, Chap. 7]. Then from the first expression in $(4.3)$ we have $\hat{s}_{\eta}(m)=0$ if and only if $\frac{\partial}{\partial R} J_{m}(k R)=0$ and $J_{m}(k R)=0$. But this is not possible, since otherwise by Green's representation theorem the function $v(r, \theta):=J_{m}(k r) e^{i m \theta}$ would be identically zero in the disk with radius $R$.

We now claim that the eigenvalues $\hat{s}_{\eta}(m)$ and their derivatives with respect to the outer disc radius $r$ decay exponentially with a rate that depends on the radius ratio of $R$ to $r$.

Lemma 4.2. For $\eta \neq 0$ and $\epsilon>0$ arbitrarily small there exist constants $c_{s}>0$ and $C_{s}>0$ such that for $m \in \mathbb{Z}$, both the following hold:

$$
\begin{gathered}
c_{s}\left(\frac{r}{R}\right)^{-|m|} \leq\left|\hat{s}_{\eta}(m)\right| \leq C_{s}\left(\frac{r}{R}\right)^{-|m|}, \\
\left|\frac{\partial}{\partial r} \hat{s}_{\eta}(m)\right| \leq C_{s}\left[\left(\frac{r}{R}\right)-\epsilon\right]^{-|m|},
\end{gathered}
$$

where $c_{s}$ and $C_{s}$ depend on $k, R$, and $r$, and $C_{s}$ additionally depends on $\epsilon$.

Proof. Consider the three terms in (4.3). Large-order asymptotics for Bessel functions [1, eq. 9.3.1] yield

$$
J_{m}(k R) \sim \frac{1}{\sqrt{2 \pi m}}\left(\frac{e k R}{2 m}\right)^{m}, \quad Y_{m}(k r) \sim-\sqrt{\frac{2}{\pi m}}\left(\frac{e k r}{2 m}\right)^{-m}
$$


for fixed $\mathrm{z}$ and $m \rightarrow \infty$. Since $H_{m}(z)=J_{m}(z)+i Y_{m}(z)$ it follows that $H_{m}(z) \sim$ $i Y_{m}(z)$, and therefore

$$
\begin{aligned}
H_{m}^{(1)}(k r) J_{m}(k R) & \sim-\frac{i}{\pi m}\left(\frac{R}{r}\right)^{m}, \\
H_{m}^{(1)}(k r) J_{m-1}(k R) & \sim-\frac{2 i}{e k R \pi}\left(\frac{R}{r}\right)^{m}, \\
H_{m}^{(1)}(k r) J_{m+1}(k R) & \sim-\frac{e k R i}{2 \pi} \frac{1}{m^{2}}\left(\frac{R}{r}\right)^{m} .
\end{aligned}
$$

Inserting these into (4.3), we get

$$
\hat{s}_{\eta}(m) \sim \frac{1}{2 e R}\left(\frac{R}{r}\right)^{m}-\frac{R e k^{2}}{8 m^{2}}\left(\frac{R}{r}\right)^{m}+\frac{i \eta}{2 m}\left(\frac{R}{r}\right)^{m} \sim \frac{1}{2 e R}\left(\frac{R}{r}\right)^{m} .
$$

Together with the reflection laws $J_{-m}(z)=(-1)^{m} J_{m}(z)$ and $H_{-m}^{(1)}(z)=(-1)^{m} H_{m}^{(1)}(z)$, the upper bound in (4.4) follows. Furthermore, by Lemma 4.1 there exists $c_{s}>0$ such that also the lower bound in (4.4) is valid. We apply similar estimates to the expression

$$
\frac{\partial}{\partial r} \hat{s}_{\eta}(m)=\frac{k \pi}{4}\left[H_{m-1}^{(1)}(k r)-H_{m+1}^{(1)}(k r)\right]\left[\frac{i k}{2}\left(J_{m-1}(k R)-J_{m+1}(k R)\right)-\eta J_{m}(k R)\right]
$$

to obtain

$$
\frac{\partial}{\partial r} \hat{s}_{\eta}(m) \sim C m\left(\frac{r}{R}\right)^{-|m|}
$$

for some constant $C \in \mathbb{C}$. The dominant contribution is $H_{m+1}^{(1)}(k r) J_{m-1}(k R)$. Absorbing the algebraic factor $m$ into the exponential bound and choosing $C_{s}$ sufficiently large yields (4.5).

The MFS is now obtained by applying to the above layer potential formulation the discrete sources ansatz $g(\phi)=\sum_{j=1}^{N} c_{j} \delta\left(\phi-\phi_{j}\right)$, where $\phi_{j}=\frac{2 \pi j}{N}, j=1, \ldots, N$. It follows that

$$
\left(S_{\eta} g\right)(\theta)=\sum_{j=1}^{N} c_{j}\left[\frac{i}{4} \frac{\partial}{\partial R} H_{0}^{(1)}\left(k\left|r e^{i \theta}-R e^{i \phi_{j}}\right|\right)-\frac{\eta}{4} H_{0}^{(1)}\left(k\left|r e^{i \theta}-R e^{i \phi_{j}}\right|\right)\right] .
$$

Note $g \notin L^{2}[0,2 \pi]$, but $S_{\eta}$ has a smooth bounded kernel so is well defined with $g$ merely integrable.

We will also need the Fourier series for this choice of $g$, which we note converges only distributionally. Denote by $\hat{c}$ the discrete Fourier transform of the coefficient vector $c=\left[c_{1}, \ldots, c_{N}\right]$ defined by

$$
\hat{c}_{s}=\frac{1}{N} \sum_{j=1}^{N} c_{j} e^{-i s \phi_{j}}, \quad-\frac{N}{2}<s \leq \frac{N}{2} .
$$

Then applying our ansatz to the definition of the Fourier coefficients,

$$
\hat{g}(m)=\frac{1}{2 \pi} \sum_{j=1}^{N} c_{j} e^{i m \phi_{j}}=\frac{N}{2 \pi} \hat{c}_{(m \bmod N)},
$$


where $m \bmod N$ denotes the unique integer lying in the range $-N / 2+1, \ldots, N / 2$ which differs from $m$ by an integer multiple of $N$. This "wrapping" of the vector $\hat{c}$ is due to the delta-comb in $g$.

Equation (4.7) is a special case of the general monopole-plus-dipole formulation (2.2), with $\Gamma_{F}$ circular and the evaluation restricted to the concentric circle $\partial D$. The basis functions in (2.2), viewed as functions on $\mathbb{R}^{2} \backslash D$, automatically satisfy the Sommerfeld radiation conditions. Thus the only remaining way to solve the boundary value problem laid out at the start of this section would be to choose the coefficients $c_{j}$ to match the boundary data $f$, e.g., by collocation or a least-squares method on $\partial D$. We remind the reader that in this work we instead couple the MFS (with the choices $D=\Omega_{e}$ and $N=N_{e}$ ) to other basis representations in $E$.

5. Convergence analysis. Let $u$ be the exact solution of (1.1)-(1.3) with given $u_{\text {inc }}$. Since $u$ and its derivative are continuous across element boundaries, we may study the convergence of approximations to $u$ independently in each subdomain. In particular, we will make use of the rate of approximation by Fourier-Bessel functions in each subdomain $E_{i}$, and the rate of approximation on the boundary $\Gamma_{e}$ by fundamental solutions. Convergence to $u$ then automatically leads to a minimization of the jump on the interfaces, as we now show. Using the triangle inequality and estimating the boundary $L^{2}$ norm by the $L^{\infty}$ norm over the whole domain, we estimate (2.4) by

$$
\begin{aligned}
J(v)= & \sum_{i<j} \int_{\Gamma_{i j}}\left|\left[\partial_{\nu} v-\partial_{\nu} u\right]\right|^{2} d s+k^{2}|[v-u]|^{2} d s \\
& +\sum_{i=1}^{q} \int_{\Gamma_{i} \cap \Gamma_{e}}\left|\left[\partial_{\nu}\left(\hat{u}_{i n c}+v-u\right)\right]\right|^{2}+k^{2}\left|\left[\hat{u}_{i n c}+v-u\right]\right|^{2} d s \\
\leq & C_{1} \sum_{i}\left\{k^{2}\left\|u_{s}-v_{e}\right\|_{L^{2}\left(\Gamma_{i} \cap \Gamma_{e}\right)}^{2}+\left\|\partial_{\nu} u_{s}-\partial_{\nu} v_{e}\right\|_{L^{2}\left(\Gamma_{i} \cap \Gamma_{e}\right)}^{2}\right\} \\
& +C_{2}\left\{k^{2} \sum_{i}\left\|u-v_{i}\right\|_{L^{\infty}\left(E_{i}\right)}^{2}+\sum_{i<j}\left\|\partial_{\nu} u-\partial_{\nu} v_{i}\right\|_{L^{\infty}\left(\Gamma_{i j}\right)}^{2}\right\},
\end{aligned}
$$

where $v \in V$ (recalling the definitions of $v_{i}, v_{e}$ from section 2), and $C_{1}, C_{2}$ are meshdependent constants.

The analysis of the rate of convergence of approximating $u$ by $v_{i} \in V_{i}$ in each subdomain $E_{i}$ is based on transforming the approximation problem involving Bessel functions into a polynomial approximation problem in the complex plane. For this it is useful to work in the $L^{\infty}$ norm. On $\Gamma_{e}$ the rate of convergence of the fundamental solutions will be analyzed using Fourier series estimates, for which the natural space is $L^{2}$.

The convergence analysis for approximating solutions of $\Delta u+\lambda u=0$ with fractional Bessel functions on domains with one corner singularity and zero Dirichlet boundary conditions at the wedges forming the singular corner was given by Betcke in [5]. The only notable new feature here is that now complex rather than real functions are approximated. This can easily be achieved by splitting the approximation problem on the subdomains $E_{i}$ into separate problems for the approximation of the real and imaginary parts of $u$.

The following theorem summarizes the results of [5, Thm. 5.2 and Lem. 5.5] adapted to our notation in this paper. We refer to [5, sect. 5] for the proof.

TheOREM 5.1. For every element $E_{i}, i=1, \ldots, q$, the following statement holds. 
There exists $\rho_{i}>1$ such that, for every $1<\tau<\rho_{i}$ and each interface $\Gamma_{i j}$ between $E_{i}$ and a neighboring subdomain $E_{j}$, it holds that

$$
\min _{v \in V_{i}}\left(\|\nabla u-\nabla v\|_{L^{\infty}\left(\Gamma_{i j}\right)}^{2}+k^{2}\|u-v\|_{L^{\infty}\left(E_{i}\right)}^{2}\right)=O\left(\tau^{-2 N_{i}}\right)
$$

as $N_{i} \rightarrow \infty$.

The rates $\rho_{i}$ may be computed; they are the conformal distance of the nearest singularity in $u$ to (a conformal map of) the domain $E_{i}[5]$.

To estimate the convergence on $\Gamma_{e}$ of the fundamental solutions approximation to the scattered field $u_{s}$, we consider (as in section 4) only the case of concentric circles. $^{2}$ The source points for the fundamental solutions are given by $\mathbf{y}_{j}=R e^{i \phi_{j}}$, $j=1, \ldots, N_{e}$, with $\phi_{j}=\frac{2 \pi j}{N_{e}}$, and the exterior circle $\Gamma_{e}$ has radius $r$. We impose $\max _{i}\left|p_{i}\right|<R<r$. The proof of the following theorem is given in section 5.1.

TheOREM 5.2. Let $\epsilon>0$ and $\tilde{\epsilon}>0$ be arbitrarily small. Define $\rho:=\min _{i} \frac{r}{\left|p_{i}\right|}$ and $\rho_{\epsilon}:=\rho-\epsilon$. Let $\nu$ be the outward normal direction at the disk with radius $r$ and assume that $N_{e}$ is even. Then

$$
\min _{v \in V_{e}}\left(\left\|\partial_{\nu} u_{s}-\partial_{\nu} v\right\|_{L^{2}\left(\Gamma_{e}\right)}^{2}+k^{2}\left\|u_{s}-v\right\|_{L^{2}\left(\Gamma_{e}\right)}^{2}\right)=\left\{\begin{array}{cc}
O\left(\left(\frac{r}{R}-\tilde{\epsilon}\right)^{-2 N_{e}}\right), & \frac{r}{R}<\rho_{\epsilon}^{\frac{1}{2}} \\
O\left(\rho_{\epsilon}^{-N_{e}}\right), & \frac{r}{R}>\rho_{\epsilon}^{\frac{1}{2}}
\end{array}\right.
$$

as $N_{e} \rightarrow \infty$.

Remark 5.3. If we consider only the rate of convergence for approximating the boundary value of $u_{s}$ by $v \in V_{e}$ and not the normal derivatives, then $\tilde{\epsilon}=0$ is possible.

Combining (5.1), Theorem 5.1, and Theorem 5.2, we immediately obtain the following.

TheOREM 5.4. Let $\rho_{1}, \ldots, \rho_{q}$ be the measures of the exponential convergence rates given in Theorem 5.1, let $\rho_{e}:=\min \left\{\left(\frac{r}{R}\right)^{2}, \min _{i} \frac{r}{p_{i}}\right\}$, and let $n_{1}, \ldots, n_{q}$ and $n_{e}$ be the multipliers for the number of basis functions as defined in (2.3). Assume that $n_{e}$ is even, and let $\omega>1$ be defined by

$$
\omega^{2}:=\min \left\{\rho_{1}^{2 n_{1}}, \ldots, \rho_{q}^{2 n_{q}}, \rho_{e}^{n_{e}}\right\} .
$$

Then

$$
\min _{v \in V} J(v)=O\left((\omega-\epsilon)^{-2 N}\right)
$$

for any $\epsilon>0$ arbitrarily small.

Remark 5.5. An ideal choice of the multipliers $n_{1}, \ldots, n_{q}$ and $n_{e}$ is such that

$$
\rho_{1}^{2 n_{1}} \approx \cdots \approx \rho_{q}^{2 n_{q}} \approx \rho_{e}^{n_{e}}
$$

This choice keeps to a minimum the number of basis functions required for a certain accuracy.

\footnotetext{
${ }^{2}$ The convergence analysis of the MFS for integral equations with periodic kernels on more general domains has recently been considered by Kangro [22]. To understand the method presented in this paper, the case of the MFS in the exterior of a disk is sufficient for many applications and can be treated completely using arguments from Fourier analysis.
} 
Remark 5.6. It is an open question whether there exists $C>0$ such that

$$
\|u-v\|_{L^{2}(E)}^{2} \leq C J(v) .
$$

In [24, Thm. 3.1] such an estimate was proved for the case that $\Omega$ is a sufficiently smooth domain and $\Gamma_{e}$ has the impedance condition $\partial_{\nu} u-i k u=0$. The crucial difference here is that we use fundamental solutions approximations instead of local absorbing boundary conditions. Similar estimates have also been investigated by Hiptmair, Moiola, and Perugia in the context of plane wave discontinuous Galerkin methods [21]. In section 6 we present numerical examples which support the conjecture (5.3).

5.1. Convergence of the MFS approximations. In this section we give a proof of Theorem 5.2. The proof proceeds along similar lines as the proof of $[4$, Thm. 3]. We proceed by finding a specific function $v \in V_{e}$, for which we show that (5.2) holds. In the following, $C>0$ will always denote an unspecified constant that may change in the course of the derivation and that depends on $k, R, r$, and $\epsilon$ but not on $N_{e}$.

Let $t[v]:=\left\|u_{s}-v\right\|_{L^{2}\left(\Gamma_{e}\right)}$ for $v \in V_{e}$. The multipole (Laurent-type) representation of $u_{s}$ is

$$
u_{s}(r, \theta)=\sum_{m \in \mathbb{Z}} \alpha_{m} H_{m}^{(1)}(k r) e^{i m \theta}
$$

for $r>\max _{i}\left|p_{i}\right|$ and $\theta \in[0,2 \pi]$. It follows immediately that the Fourier coefficients $\hat{u}_{s}(m)$ of the angular function $u_{s}(r, \cdot)$ are

$$
\hat{u}_{s}(m)=\alpha_{m} H_{m}^{(1)}(k r) .
$$

The following lemma gives a bound on the decay of $\hat{u}_{s}(m)$ as $m \rightarrow \infty$.

Lemma 5.7. Let $\rho:=\min _{i} \frac{r}{\left|p_{i}\right|}$ and $\rho_{\epsilon}:=\rho-\epsilon$. Then

$$
\left|\hat{u}_{s}(m)\right| \leq C \rho_{\epsilon}^{-|m|}
$$

for any arbitrarily small $\epsilon>0$, where $C$ depends on $k, R, r$, and $\epsilon$.

Proof. Let $f(t):=u_{s}(r, 2 \pi t)$ for $t \in[0,1]$. We need to show that $f$ can be analytically continued as a holomorphic function to the strip $t+i \tau$ for $|\tau|<\frac{1}{2 \pi} \log \rho$. Denote by $\tilde{p}:=\arg \max _{i}\left|p_{i}\right|$ the corner of $\partial \Omega$ with largest absolute value. Reparameterize the scattered field $u_{s}(x, y)$ in the Cartesian $(x, y)$ coordinates as $U_{s}(z, \bar{z}):=u_{s}(x, y)$, where $z=x+i y$. Let $\Omega_{\tilde{p}}:=\left\{z:|\tilde{p}|<|z|<\frac{r^{2}}{|\tilde{p}|}, \operatorname{Im}\{z\}>0\right\}$ be a half annulus, and define $\Omega_{\tilde{p}}^{*}:=\left\{z: \bar{z} \in \Omega_{\tilde{p}}\right\}$. By a result from Vekua [19,28], $U_{s}$ can be analytically continued as a holomorphic function of two independent complex variables $z$ and $z^{*}$ into the whole of $\Omega_{\tilde{p}} \times \Omega_{\tilde{p}}^{*}$, such that $U_{s}\left(z, z^{*}\right)=u_{s}(x, y)$ if and only if $z^{*}=\bar{z}$. From the definition of $f$ it follows that $f(t)=U_{s}\left(r e^{i 2 \pi t}, r e^{-i 2 \pi t}\right)$. Hence, by the analyticity of $U_{s}$ we have that $f(t+i \tau)=U_{s}\left(r e^{-2 \pi \tau+i 2 \pi t}, r e^{2 \pi \tau-i 2 \pi t}\right)$ is analytic for $0<t<1 / 2$ and $-\frac{1}{2 \pi} \log \rho<\tau<\frac{1}{2 \pi} \log \rho$. By rotating the half annulus and repeating the argument, the analyticity in this strip can be extended to the whole interval $t \in[0,1]$. The estimate (5.4) now follows from the decay of Fourier coefficients of analytic functions (see, for example, [20, sect. 13.2]).

The Fourier series representation of $v \in V_{e}$, associated with density $g$, restricted to the circle of radius $r$ is given by (see section 4 )

$$
\hat{v}(m)=\widehat{S_{\eta} g}(m)=\hat{s}_{\eta}(m) \hat{g}(m),
$$


where $g(\theta)=\sum_{j=1}^{N_{e}} c_{j} \delta\left(\phi-\phi_{j}\right)$ and $\hat{g}(m)=\frac{N_{e}}{2 \pi} \hat{c}_{\left(m \bmod N_{e}\right)}$.

By reparameterizing the $L^{2}$ integral over $\Gamma_{e}$ and using Parseval's identity, it follows that

$$
t[v]^{2}=2 \pi r \sum_{m \in \mathbb{Z}}\left|\hat{u}_{s}(m)-\hat{v}(m)\right|^{2} .
$$

We now take a specific $v \in V_{e}$ by choosing the MFS coefficients of $v$ such that for all $-N_{e} / 2<m \leq N_{e} / 2$, we have

$$
\hat{g}(m)=\frac{\hat{u}_{s}(m)}{\hat{s}_{\eta}(m)}=\frac{\alpha_{m} H_{m}^{(1)}(k r)}{\frac{\pi}{2} H_{m}^{(1)}(k r)\left[\frac{i k}{2}\left(J_{m-1}(k R)-J_{m+1}(k R)\right)-\eta J_{m}(k R)\right]} .
$$

Then the terms involving $-N_{e} / 2<m \leq N_{e} / 2$ cancel out in (5.5) and we can estimate

$t[v]^{2} \leq C\left(\sum_{m \notin\left[-\frac{N_{e}}{2}+1, \ldots, \frac{N_{e}}{2}\right]}\left|\hat{u}_{s}(m)\right|^{2}+\sum_{m \notin\left[-\frac{N_{e}}{2}+1, \ldots, \frac{N_{e}}{2}\right]}\left|\hat{s}_{\eta}(m) \hat{g}(m)\right|^{2}\right)=: C\left(E_{u}^{2}+E_{s}^{2}\right)$.

Using (5.4) we can now estimate $E_{u}^{2}$ as

$$
E_{u}^{2} \leq C \sum_{m \notin\left[-\frac{N_{e}}{2}+1, \ldots, \frac{N_{e}}{2}\right]} \rho_{\epsilon}^{-2|m|} \leq C \rho_{\epsilon}^{-N_{e}} .
$$

In order to estimate $E_{s}^{2}$ we rewrite it as

$$
E_{s}^{2}=\sum_{n=-\frac{N_{e}}{2}+1}^{\frac{N_{e}}{2}} \hat{g}(n)^{2} \sum_{b \in \mathbb{Z} \backslash\{0\}} \hat{s}_{\eta}\left(b N_{e}+n\right)^{2} .
$$

Bounding the inner sum and substituting $|\hat{g}(n)|=\left|\frac{\hat{u}_{s}(n)}{\hat{s}_{\eta}(n)}\right| \leq C\left(\frac{r}{R \rho_{\epsilon}}\right)^{|n|}$ gives

$$
E_{s}^{2} \leq C \sum_{n=-\frac{N_{e}}{2}+1}^{\frac{N_{e}}{2}}|\hat{g}(n)|^{2}\left(\frac{r}{R}\right)^{-2 N_{e}+2|n|} \leq C\left(\frac{r}{R}\right)^{-2 N_{e}} \sum_{n=-\frac{N_{e}}{2}+1}^{\frac{N_{e}}{2}}\left(\frac{r^{2}}{R^{2} \rho_{\epsilon}}\right)^{2|n|}
$$

If $\rho_{\epsilon}>\frac{r^{2}}{R^{2}}$, it follows from (5.8) that $E_{s}^{2} \leq C\left(\frac{r}{R}\right)^{-2 N_{e}}$. This decays slower than the estimate in (5.7). Hence, we have $t[v]^{2} \leq C\left(\frac{r}{R}\right)^{-2 N_{e}}$. If $\rho_{\epsilon}<\frac{r^{2}}{R^{2}}$, it follows from (5.8) that

$$
E_{s}^{2} \leq C\left(\frac{r}{R}\right)^{-2 N_{e}}\left(\frac{r^{2}}{R^{2} \rho_{\epsilon}}\right)^{N_{e}}=C \rho_{\epsilon}^{-N_{e}},
$$

which has the same exponential rate of decay as (5.7), and therefore $t[v]^{2} \leq C \rho_{\epsilon}^{-N_{e}}$ in this case.

We now show that up to an arbitrarily small correction in rate $\tilde{\epsilon}>0$ the error $t^{\prime}[v]:=\left\|\partial_{\nu} u_{s}-\partial_{\nu} v\right\|_{L^{2}\left(\Gamma_{e}\right)}$ has the same exponential bounds as $t[v]$. Similarly to (5.5) we have

$$
t^{\prime}[v]=2 \pi r \sum_{m \in \mathbb{Z}}\left|\widehat{\partial_{\nu} u_{s}}(m)-\widehat{\partial_{\nu} v}(m)\right|^{2}
$$


where

$$
\widehat{\partial_{\nu} u_{s}}(m)=\frac{\partial}{\partial r} \hat{u}_{s}(m)
$$

and

$$
\widehat{\partial_{\nu} v}(m)=\frac{\partial}{\partial r} \hat{v}(m)=\left(\frac{\partial}{\partial r} \hat{s}_{\mu}(m)\right) \hat{g}(m)
$$

The second equality follows from the fact that $\hat{g}(m)$ is independent of $r$ (see (5.6)). Furthermore, from (5.6) it follows that

$$
\frac{\frac{\partial}{\partial r} \hat{u}_{s}(m)}{\frac{\partial}{\partial r} \hat{s}_{\mu}(m)}=\frac{\hat{u}_{s}(m)}{\hat{s}_{\eta}(m)}=\hat{g}(m)
$$

for $-N / 2<m \leq N / 2$. Hence, the Fourier coefficients involving $-N / 2<m \leq N / 2$ also cancel out for $t^{\prime}[v]$. By Lemma $4.2, \frac{\partial}{\partial r} \hat{s}_{\eta}(m)$ has, up to an arbitrarily small rate correction $\tilde{\epsilon}$, the same exponential upper bound as $\hat{s}_{\eta}(m)$. Also, since $\frac{\partial}{\partial r} u_{s}$ has its only possible singularities at the corners $p_{i}$, its Fourier coefficients $\frac{\partial}{\partial r} \hat{u}_{s}(m)$ have the same exponential bound (5.4) as those of $u_{s}$. We can therefore apply the same arguments to estimate $t^{\prime}[v]$ as we did for $t[v]$ and arrive (up to $\tilde{\epsilon}$ coming from the bound on $\frac{\partial}{\partial r} \hat{s}_{\eta}(m)$ ) at the same exponential bounds as for $t[v]$, which concludes the proof of Theorem 5.2.

6. Numerical examples. In this section we demonstrate the numerical properties of the method and present computational results for scattering on a square, a snowflake domain, and a cavity. All computations were performed using MATLAB 2009a on a dual processor workstation with two $2.8 \mathrm{GHz}$ Intel Xeon quad-core processors and 64GB RAM. However, the MATLAB multithreading rarely utilized more than one $\mathrm{CPU}$ core. Hence, the timings will be comparable on a standard desktop machine.

6.1. Scattering from a square. Let $\Omega$ be the unit square given by $\Omega=$ $(-0.5,0.5)^{2}$. We use a domain decomposition as shown in Figure 2.1. $\Gamma_{e}$ is a circle with radius $r$, and the source points for the fundamental solutions are given by $\mathbf{y}_{j}=R e^{i \phi_{j}}, \phi_{j}=\frac{2 \pi j}{N_{e}}, j=1, \ldots, N_{e}$. We found that the fundamental solutions basis size $N_{e}:=2 N$ was sufficient, where $N$ is the number of Fourier-Bessel sine functions in each element $E_{i}$. For the fundamental solutions we use the combined formulation (2.2) with $\eta=k$. For quadrature we used the Clenshaw-Curtis rule with a number of nodes chosen high enough that further increases had negligible effect; typically this number is a small multiple of $N$ per interface $\Gamma_{i j}$. (Code for this setup is given in Appendix A.)

Rate of convergence. In Figure 6.1 we show the rate of convergence of $t\left[v_{L S}\right]:=$ $J\left(v_{L S}\right)^{1 / 2}$, which measures the norm of the jumps in function values and normal derivatives on the interfaces. We fix $R=0.8$ and the wavenumber $k=1$. The left plot shows the case $r=0.9$ and the right plot $r=1.3$. The measured convergence (solid-dotted line) for the latter is much faster than for the former. In both cases we have also plotted the asymptotic bound $\omega^{-N}$ from Theorem 5.4. The asymptotic estimates for the convergence on the finite elements $E_{1}, \ldots, E_{4}$ can be computed using numerical conformal mapping techniques (see [5] for more details). The convergence of the MFS approximations is directly given by Theorem 5.2. 
What is the optimal value of $r$ ? If $r$ is small, then the singular corners of the square have a large relative distance to the neighboring elements, suggesting a fast convergence on these elements. However, the rate of convergence for the MFS approximations will be slow since the radius $r$ is close to the radius of the corners of the polygon. If $r$ is large, then the MFS approximations converge fast but the relative distance of a singular corner to a neighboring element $E_{i}$ is very small, leading to slow convergence on the finite elements. This suggests that there is an optimal radius $r=r_{\text {opt }}$, which is achieved when the asymptotic rate of convergence of the MFS is identical to the asymptotic rate of convergence on the finite elements.

In the left plot of Figure 6.2 we compute (using conformal mapping) the asymptotic exponential convergence factor $\omega$ from Theorem 5.4 as a function of the radius $r$ of the outer circle $\Gamma_{e}$. The optimum asymptotic rate is achieved for $r \approx 1.036$. In that case the rates of convergence on the finite elements $E_{j}$ and on $\Omega_{e}^{+}$are almost identical, with a rate of approximately $1.465^{-N}$. The right plot of Figure 6.2 shows the convergence of $t\left[v_{L S}\right]$ in this case. For $N=80$ the value of $t\left[v_{L S}\right]$ is close to machine precision.

Sound-hard boundary conditions. In this paper we have analyzed the case of sound-soft boundary conditions for $u$, that is, $u=0$ on $\partial \Omega$. However, the scheme presented in this paper can be trivially extended to sound-hard boundary conditions, that is, $\partial_{\nu} u=0$, where $\partial_{\nu}$ denotes the outward pointing normal derivative on $\partial \Omega$. For this we switch from Fourier-Bessel sine function approximations to Fourier-Bessel cosine function approximations in each $E_{i}$, replacing (2.1) by

$$
u(r, \theta) \approx \sum_{j=0}^{N_{i}} c_{j}^{(i)} J_{j \alpha_{i}}(k r) \cos j \alpha_{i} \theta .
$$

It is simple to check that these functions satisfy the homogeneous boundary conditions for the normal derivative. The fundamental solutions basis functions in $\Omega_{e}^{+}$do not change. This is a great advantage compared to combined integral equation formulations, which usually involve the approximation of a hypersingular integral operator for the sound-hard scattering case.

Timing results. In Figure 6.3 we show the real part of the full field for the soundsoft scattering case (left plot) and the sound-hard scattering case (right plot) for $k=50$, i.e., about 8 wavelengths on a side. The incident field is a plane wave with direction $-\frac{\pi}{6}$.

The solution time was approximately 11 seconds in both cases. This includes the setup of the problem and the time for solving the least-squares problem $\min _{c} \| W(A c-$ b) $\|_{2}$, where $A$ is of dimension $4816 \times 600$ (sound-soft case), or $4816 \times 604$ (sound-hard case), corresponding to $N=100$ and $m_{i j}=200$ Clenshaw-Curtis quadrature points on each interface $\Gamma_{i j}$. In the sound-soft case we have $t\left[v_{L S}\right] \approx 1.3 \times 10^{-10}$ and in the sound-hard case $t\left[v_{L S}\right] \approx 9.2 \times 10^{-11}$. Creating the solution plots takes longer, since the solution needs to be evaluated on a large number of grid points.

6.2. Scattering from a snowflake domain. We now show results for a snowflake domain, which is nonconvex but star-shaped. The domain and the corresponding decomposition are shown in the top two plots of Figure 6.4. Note that the external boundary $\Gamma_{E}$ is not a circle but an equipotential line of the exterior (Laplace) Green's function of the domain. Although we have given the analysis only for $\Gamma_{E}$ being a circle, we demonstrate in this example that domain-adapted analytic curves also work well. 

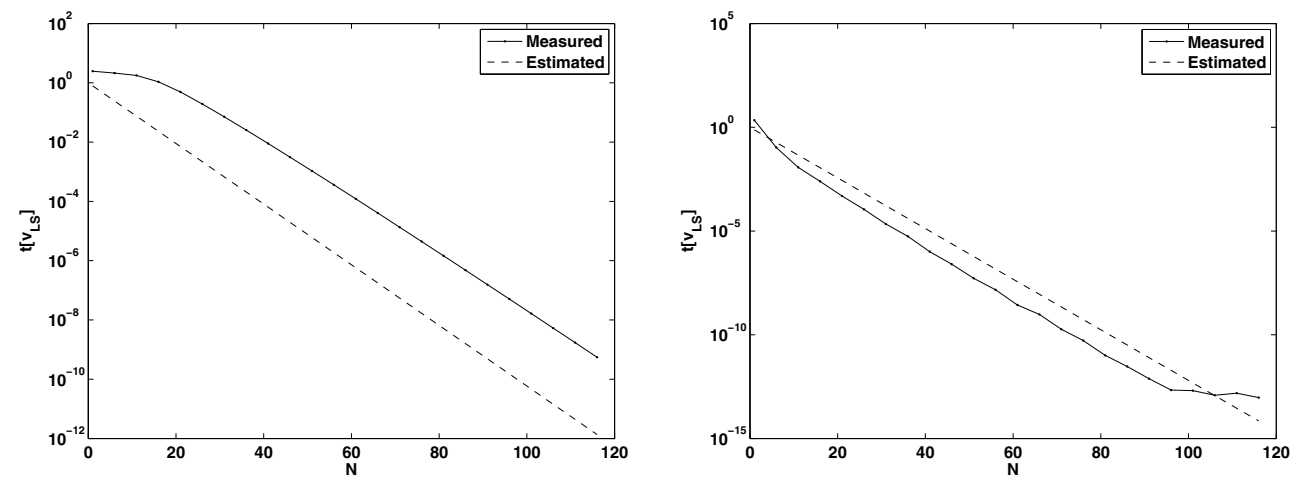

FiG. 6.1. Left plot: Convergence of $t\left[v_{L S}\right]$ (solid-dotted line) for growing $N$ for the case $r=0.9$ compared to the estimated rate of convergence from Theorem 5.4 (dashed line). Right plot: Same as left plot but now with $r=1.3$. The convergence is much faster for this choice of $r$.
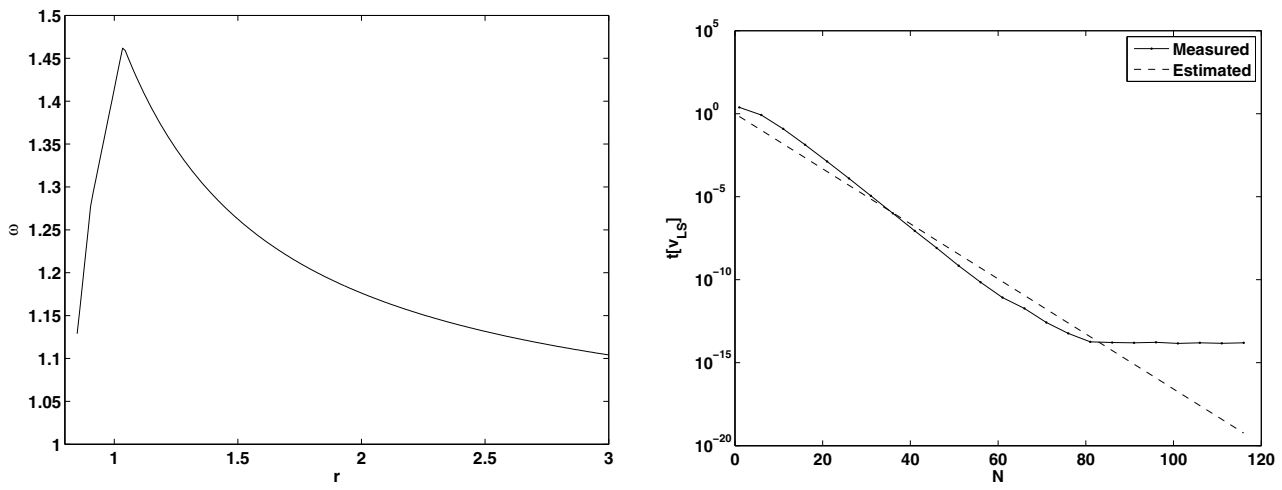

FIG. 6.2. Left plot: Asymptotic convergence factor $\omega$ in dependence of $r$. Large values of $\omega$ indicate faster convergence of $t\left[v_{L S}\right]$. Right plot: Measured (solid-dotted line) and estimated (dashed line) convergence for the optimal radius $r=1.036$.
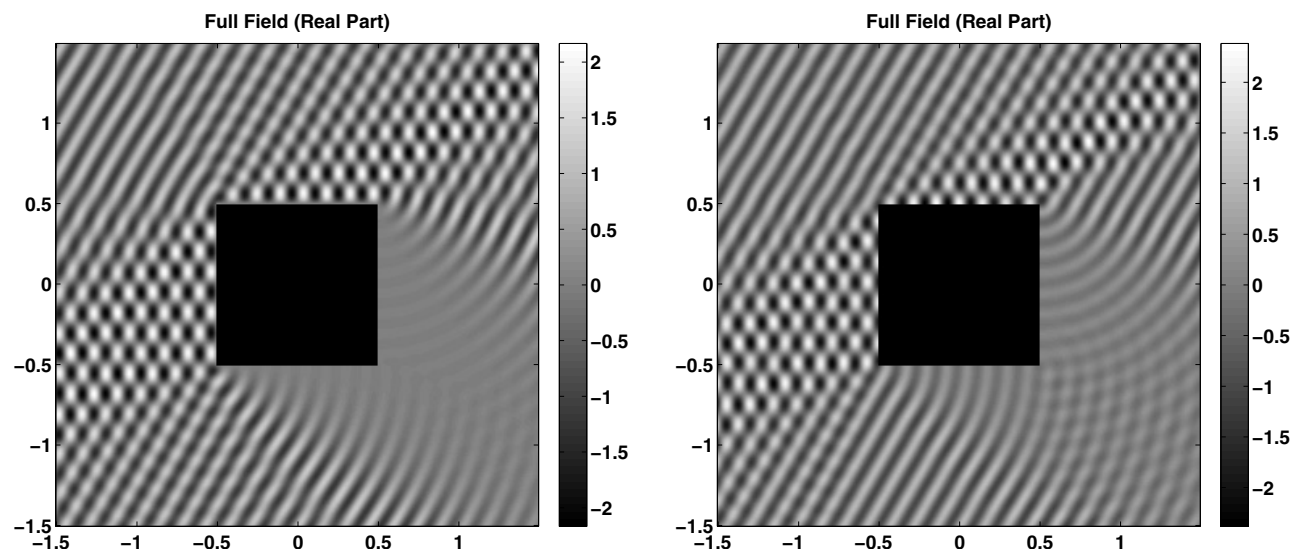

FIG. 6.3. Real part of the full field for sound-soft scattering on the square (left plot) and soundhard scattering on the square (right plot). In both cases $k=50$ and the incident wave is a plane wave with angle $-\frac{\pi}{6}$. 
TABLE 6.1

Results for different wavenumbers for sound-soft scattering on the snowflake domain.

\begin{tabular}{c|c|c|c|c|c}
\hline$k$ & $N$ & $m$ & $N_{V}$ & $t\left[v_{L S}\right]$ & Time \\
\hline 50 & 70 & 1980 & 1071 & $3 \cdot 10^{-8}$ & $8 \mathrm{~s}$ \\
100 & 90 & 2460 & 1377 & $4 \cdot 10^{-9}$ & $15 \mathrm{~s}$ \\
200 & 130 & 3660 & 1989 & $5 \cdot 10^{-9}$ & $44 \mathrm{~s}$ \\
500 & 260 & 8700 & 3978 & $2 \cdot 10^{-7}$ & $7 \mathrm{~m}$ \\
\hline
\end{tabular}

In the bottom two plots of Figure 6.4 we show the scattered field (left) and the full field (right) for sound-soft scattering by an incident plane wave with angle $-\frac{\pi}{3}$ and wavenumber $k=100$. Setting up and solving the dense least-squares problem takes 15 seconds. The error $t\left[v_{L S}\right]$ is approximately $4 \cdot 10^{-9}$.

In Table 6.1 we show results for different wavenumbers. $N$ was chosen such that the error $t\left[v_{L S}\right]$ is in the approximate range $10^{-7}$ to $10^{-9}$. The number of fundamental solutions used was $N_{e}=3.3 \mathrm{~N} . \mathrm{m}$ and $N_{V}$ denote the dimensions of the matrix $A \in \mathbb{C}^{m \times N_{V}}$ in the least-squares problem $\min _{c}\|W(A c-b)\|_{2}$. The table lists the total times for setting up the matrix and solving the least-squares problem. Up to around $k=200$ the solution time is excellent. For higher $k$ the $O\left(k^{3}\right)$ dependence of the solution time becomes a problem. Also at this high wavenumber, we have noticed that the best achievable $t\left[v_{L S}\right]$ seems to be around $10^{-7}$. By contrast, Figure 6.5 shows that, at lower $k$, accuracies approaching machine precision are achievable. This figure also shows that a small increase in the number of basis functions leads to a large decrease of $t\left[v_{L S}\right]$, once the number of basis functions is high enough to get into the regime of exponential convergence. From our experiments the number of basis functions required to enter this regime grows approximately linearly with $k$, as supported by Figure 6.5. Once this regime is entered, the convergence rate appears to be independent of $k$, as suggested by the analysis in section 5 .

In Remark 5.6 we stated the conjecture that $\|u-v\|_{L^{2}(E)}^{2} \leq C J(v)$ for some $C>0$. To support this conjecture we also plotted in Figure 6.5 the convergence of the scattered field $u_{s}$. This was done by first computing an approximate solution $u_{s}$ for $N=100$ and then approximating the $L^{\infty}$ error $\left\|u_{s}^{(N)}-u_{s}\right\|_{L^{\infty}\left(\mathbb{R}^{2} \backslash \Omega\right)}$, where $u_{s}^{(N)}$ is the approximate scattered field in step $N$, by evaluating it on the same grid that was used to plot the solution in Figure 6.4. Notice that the $L^{\infty}$ errors are always at least a factor of 50 smaller than $t\left[v_{L S}\right]$. Therefore this plot supports the stronger conjecture

$$
\left\|u-\hat{u}_{i n c}-v\right\|_{L^{\infty}\left(\mathbb{R}^{2} \backslash \Omega\right)}^{2} \leq C J(v),
$$

where $\hat{u}_{i n c}$ is as defined in (2.5) and $C>0$ is a constant, which is, however, expected to be large if exterior resonance effects occur.

6.3. A cavity domain. To conclude this section we demonstrate the example of scattering from a non-star-shaped cavity domain, which allows strong resonances to occur. The domain decomposition is shown in the left plot of Figure 6.6. Note that the leftmost element does not share a corner with the cavity domain; in this element we use a regular Fourier-Bessel expansion, that is, (2.1) with $\alpha_{i}=1$ and both sin and cos terms, whose origin is chosen inside the element. The reason for using this new type of element is to reduce the size of the elements at the corners of the entrance to the cavity, which increases the relative distance to neighboring singularities and thereby increases their rate of exponential convergence. Even though an extra basis set is required, at high accuracy the total basis size is reduced, and hence efficiency is improved. The previous convergence analysis would easily extend to this case. 

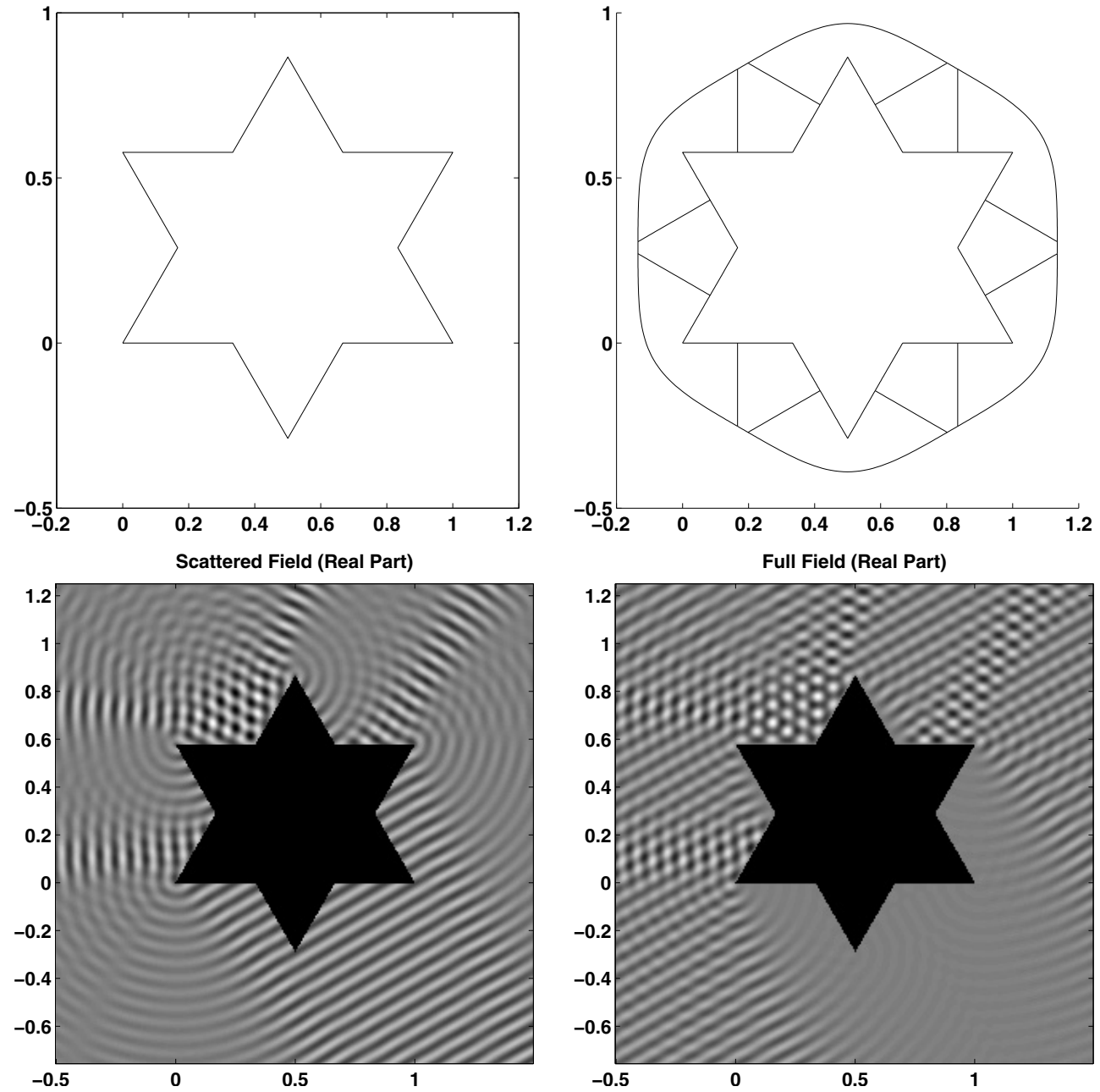

Fig. 6.4. Top plots: A snowflake-shaped domain and the corresponding domain decomposition. Bottom plots: The scattered (left) and full (right) fields for sound-soft scattering on the snowflake domain with $k=100$.
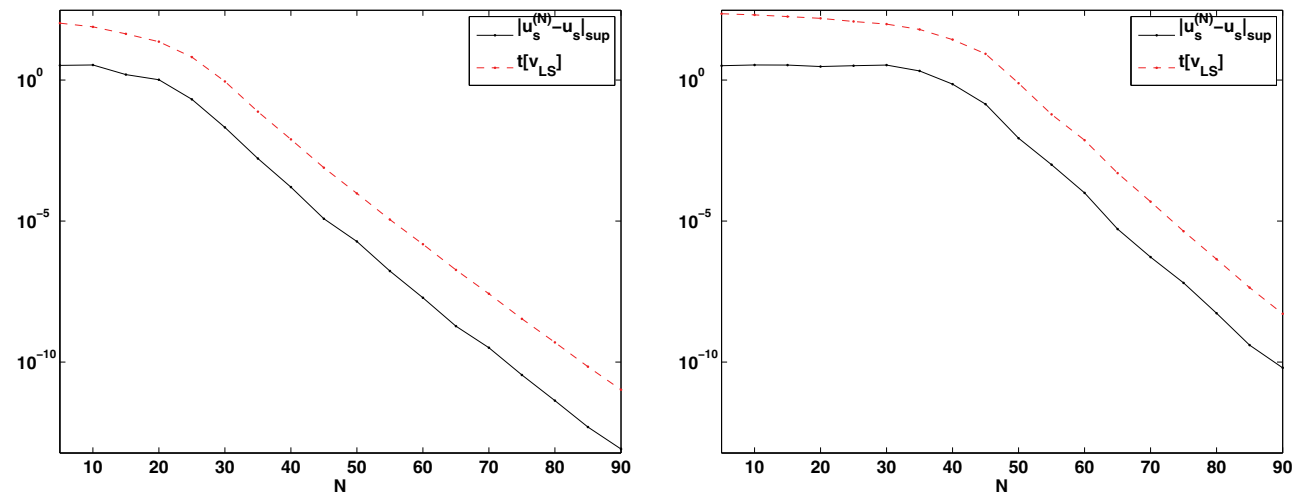

Fig. 6.5. Convergence of $t\left[v_{L S}\right]$ (dashed line) for $k=50$ (left plot) and $k=100$ (right plot). The plots also show an approximation to the convergence versus $N$ of the $L^{\infty}$ norm of the approximate scattered field $u_{s}^{(N)}$ to the exact scattered field $u_{s}$ (solid line). 

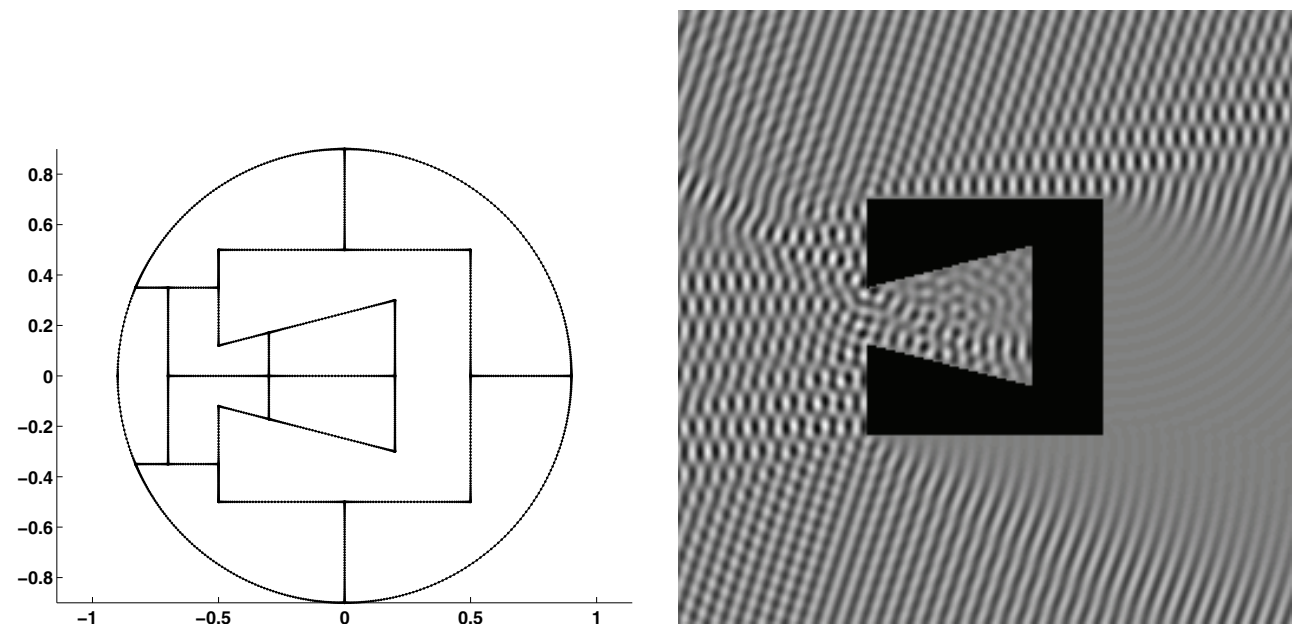

FIG. 6.6. Sound-soft scattering from a cavity domain.

In the right plot of Figure 6.6 we show the solution of sound-soft scattering by an incoming plane wave with direction $-\pi / 10$ for $k=100$, i.e., 16 wavelengths on the longest edge. The setup and solution of the associated least-squares problem takes 14 seconds (matrix size $1938 \times 1521$ ) for an error of $t\left[v_{L S}\right] \approx 3 \cdot 10^{-8}$. To evaluate and plot the solution on $6 \cdot 10^{4}$ points (a square grid of spacing 0.01 ) takes an additional 46 seconds.

7. Numerical stability of the method. The method discussed in this paper requires the solution of least-squares problems of the form (3.2), where $A \in$ $\mathbb{C}^{m \times N_{V}}$ is highly ill-conditioned and $W$ is a diagonal matrix containing quadrature weights. Sources of the ill-conditioning in $A$ are the large dynamic range of the Fourier-Bessel basis functions and the ill-conditioning of fundamental solutions bases. The ill-conditioning of the Fourier-Bessel functions can be improved by rescaling these functions so that they have unit value at the subdomain's maximum radius from the corner, which is what we have done in all example calculations. By contrast, illconditioning of the fundamental solutions is inextricably linked to the fast exponential decay of the eigenvalues $\hat{s}_{\eta}(m)$ of the associated layer potential operator $S_{\eta}$, and thus cannot be removed by scaling.

Denote by $c_{L S}$ the solution of the least-squares problem (3.2). If we have sufficiently many quadrature points, then $t\left[v_{L S}\right]$ is well approximated by the discrete error $t_{d}\left[v_{L S}\right]:=\left\|W\left(A c_{L S}-b\right)\right\|_{2}$. Solving (3.2) using a backward stable least-squares solver-such as mldivide in MATLAB - returns a solution $\tilde{c}_{L S}$, which is the exact minimizer of

$$
\min _{c \in \mathbb{C}^{N_{V}}}\|(W A+E) c-(W b+f)\|_{2},
$$

where $E$ and $f$ are small perturbations bounded by

$$
\|E\|_{2},\|f\|_{2} \leq C \epsilon_{\text {mach }}
$$

where the constant $C>0$ is small and the machine precision is $\epsilon_{\text {mach }}$ [2]. Because of ill-conditioning, $\tilde{c}_{L S}$ may differ drastically from $c_{L S}$. However, the following shows 
that the corresponding discrete least-squares functional can deviate from its true minimum only under certain conditions.

LEMma 7.1. Let $\tilde{c}_{L S}$ be defined as above, and let $\tilde{v}_{L S}$ be the corresponding solution field. Then,

$$
t_{d}\left[v_{L S}\right] \leq t_{d}\left[\tilde{v}_{L S}\right] \leq t_{d}\left[v_{L S}\right]+C\left(2+\left\|c_{L S}\right\|_{2}+\left\|\tilde{c}_{L S}\right\|_{2}\right) \epsilon_{m a c h} .
$$

Proof. Using the property that $c_{L S}$ minimizes the unperturbed least-squares problem, and also using the triangle inequality, results in

$$
\begin{aligned}
\left\|W A c_{L S}-W b\right\|_{2} & \leq\left\|W A \tilde{c}_{L S}-W b\right\|_{2} \\
& \leq\left\|(W A+E) \tilde{c}_{L S}-(W b+f)\right\|_{2}+C\left(1+\left\|\tilde{c}_{L S}\right\|_{2}\right) \epsilon_{\text {mach }} .
\end{aligned}
$$

Exchanging perturbed and unperturbed quantities in the above gives similarly

$$
\left\|(W A+E) \tilde{c}_{L S}-(W b+f)\right\|_{2} \leq\left\|W A c_{L S}-W b\right\|_{2}+C\left(1+\left\|c_{L S}\right\|_{2}\right) \epsilon_{\text {mach }} .
$$

Combining the two estimates gives (7.2).

Thus, if the basis coefficient 2-norms at the approximate and exact minima are small, the numerical least-squares solution must converge at the same exponential rate (Theorem 5.4) as the exact least-squares solution. To rigorously apply this lemma we need bounds on the norms of $\left\|c_{L S}\right\|_{2}$ and $\left\|\tilde{c}_{L S}\right\|_{2}$. A partial result towards deriving such bounds is given in the following theorem, which establishes under which conditions on $\Gamma_{F}$ the coefficients of the fundamental solutions basis functions in $\Omega_{e}$ are bounded independently of $N$. A similar result for approximating interior Helmholtz problems with fundamental solutions was given in [4].

THEOREM 7.2. Consider a sequence of fundamental solutions approximations, each of the form $v(\boldsymbol{x})=\sum_{j=1}^{N_{e}} c_{j}^{(e)}\left(\frac{i}{4} \frac{\partial}{\partial \nu\left(\boldsymbol{y}_{j}\right)} H_{0}^{(1)}\left(k\left|\boldsymbol{x}-\boldsymbol{y}_{j}\right|\right)-\frac{\eta}{4} H_{0}^{(1)}\left(k\left|\boldsymbol{x}-\boldsymbol{y}_{j}\right|\right)\right)$, with growing numbers $N_{e}$ ( $N_{e}$ even) of charge points, that attains the error bound from Theorem 5.2 as $N_{e} \rightarrow \infty$. Let each coefficient vector be written as $c^{(e)}:=\left[c_{1}^{(e)}, \ldots, c_{N_{e}}^{(e)}\right]^{T}$. If $R>\max _{i}\left|p_{i}\right|$, then the sequence of norms $\left\|c^{(e)}\right\|_{2}$ is bounded independently of $N_{e}$.

Proof. In the following, $C>0$ denotes an unspecified constant that depends on $k, R, r$, and $\epsilon$ but not on $N_{e}$, and may change throughout the proof. By assumption $\max _{i}\left|p_{i}\right|<R<r$.

Let $g(\theta)=\sum_{j=1}^{N_{e}} c_{j}^{(e)} \delta\left(\phi-\phi_{j}\right)$ and let $\hat{g}(m)=\frac{N_{e}}{2 \pi} \hat{c}_{\left(m \bmod N_{e}\right)}^{(i)}$ be the Fourier coefficients of $g$ (see section 5.1). The proof follows by showing that $\hat{g}(m)$ is bounded for $m$ in the interval $\left[-N_{e} / 2+1, \ldots, N_{e} / 2\right]$ and by applying the discrete version of Parseval's identity. For the specific choice of the coefficients $c^{(e)}$, such that $\hat{g}(m)=$ $\frac{\hat{u}_{s}(m)}{\hat{s}_{\eta}(m)}$, which was used for the convergence analysis in section 5.1, boundedness already follows for sufficiently small $\epsilon$ from

$$
|\hat{g}(m)| \leq C\left(\frac{r}{R \rho_{\epsilon}}\right)^{|m|}
$$

and the assumption $R>\max _{i}\left|p_{i}\right|$.

To establish boundedness of $|\hat{g}(m)|$ for any sequence $c^{(e)}$ that attains the optimal rate of convergence from Theorem 5.2 we proceed as follows. From (5.2) and (5.5) we have that

$$
k \sqrt{2 \pi r}\left|\hat{u}_{s}(m)-\hat{s}_{\eta}(m) \hat{g}(m)\right| \leq C \tau^{-N_{e}}, \quad m \in \mathbb{N},
$$


where $\tau=\min \left\{\left(\frac{r}{R}\right), \rho_{\epsilon}^{1 / 2}\right\}$ (note that by Remark 5.3, $\tilde{\epsilon}=0$ in Theorem 5.2 is possible since we use only the estimate for the function and not for the normal derivative). It follows that

$$
|\hat{g}(m)| \leq\left|\hat{s}_{\eta}(m)\right|^{-1}\left(C \tau^{-N_{e}}(2 \pi r)^{-1 / 2}+\left|\hat{u}_{s}(m)\right|\right), \quad m \in \mathbb{N} .
$$

Now restrict $m$ to the interval $\left[-N_{e} / 2+1, \ldots, N_{e} / 2\right]$. If $R>\sqrt{r \max _{i}\left|p_{i}\right|}$, then for sufficiently small $\epsilon$ it follows that $\tau=\frac{r}{R}$. Using (4.4) and (5.4) we have

$$
|\hat{g}(m)| \leq C\left(\frac{r}{R}\right)^{|m|}\left[\left(\frac{r}{R}\right)^{-N_{e}}+\rho_{\epsilon}^{-|m|}\right] \leq C\left(\frac{r}{R}\right)^{|m|}\left[\left(\frac{r}{R}\right)^{-|m|}+\rho_{\epsilon}^{-|m|}\right]
$$

since $r>R$ and $|m| \leq \frac{N_{e}}{2}$. From $\max _{i}\left|p_{i}\right|<R$ it follows for sufficiently small $\epsilon$ that $\rho_{\epsilon}^{-|m|} \leq\left(\frac{r}{R}\right)^{-|m|}$. Inserting this into (7.3) gives

$$
|\hat{g}(m)| \leq C,
$$

where all constants are absorbed into $C$. If $R<\sqrt{r \max _{i}\left|p_{i}\right|}$, then for sufficiently small $\epsilon$ it holds that $\tau=\rho_{\epsilon}$. We obtain, absorbing constants into $C$,

$$
\begin{aligned}
|\hat{g}(m)| & \leq C\left(\frac{r}{R}\right)^{|m|}\left[\rho_{\epsilon}^{-N_{e} / 2}+\rho_{\epsilon}^{-|m|}\right] \\
& \leq C\left(\frac{r}{R}\right)^{|m|}\left[\rho_{\epsilon}^{-|m|}+\rho_{\epsilon}^{-|m|}\right] \leq C\left(\frac{r}{R \rho_{\epsilon}}\right)^{|m|} \leq C
\end{aligned}
$$

for $\epsilon$ sufficiently small, since $\max _{i}\left|p_{i}\right|<R$, and therefore $\rho_{\epsilon}>\frac{r}{R}$.

From (4.8) it follows that $\hat{g}(m)=\frac{N_{e}}{2 \pi} \hat{c}^{(e)}(m)$ for $m \in\left[-N_{e} / 2+1, \ldots, N_{e} / 2\right]$, where the vector $\hat{c}^{(e)} \in \mathbb{C}^{N_{e}}$ is the discrete Fourier transform of $c^{(e)}$. Hence, using the boundedness of $\hat{g}(m)$ for $m \in\left[-N_{e} / 2+1, \ldots, N_{e} / 2\right]$ together with the discrete version of Parseval's identity $\left\|c^{(e)}\right\|_{2}^{2}=N_{e}\left\|\hat{c}^{(e)}\right\|_{2}^{2}$, we obtain

$$
\left\|c^{(e)}\right\|_{2}^{2}=N_{e}\left\|\hat{c}^{(e)}\right\|_{2}^{2}=\frac{(2 \pi)^{2}}{N_{e}} \sum_{j=-\frac{N_{e}}{2}+1}^{\frac{N_{e}}{2}}|\hat{g}(m)|^{2} \leq(2 \pi)^{2} C^{2},
$$

which concludes the proof.

Hence, the coefficient vector of the MFS approximations stays bounded in norm as long as $\max _{i}\left|p_{i}\right|<R$. In [4] this was studied numerically for interior Helmholtz problems, in the general case of analytic MFS and boundary curves. In that work we also showed that a large coefficient norm forces a corresponding loss of accuracy in solution evaluation due to round-off error.

Consider again the problem of sound-soft scattering on the square (section 6.1). We have $\left|p_{i}\right|=\frac{\sqrt{2}}{2}$ for all corners of the square $\Omega=(-1 / 2,1 / 2)^{2}$. If the radius of the source points $\mathbf{y}_{j}$ is $R=0.4$, then for $k=100$ and $N=150$ we find that the computed coefficient vector $\tilde{c}_{L S}$ has $\left\|\tilde{c}_{L S}\right\| \approx 3 \cdot 10^{9}$. The measured boundary error $t_{d}\left[\tilde{v}_{L S}\right] \approx 3.6$ is terrible. On the other hand, if $R=0.8>\max _{i}\left|p_{i}\right|$, then $\left\|\tilde{c}_{L S}\right\| \approx 6.2$, and $t_{d}\left[\tilde{v}_{L S}\right] \approx 7 \cdot 10^{-10}$ is excellent. Note that in both cases the matrix $W A$ is numerically singular with a condition number of around $10^{20}$.

To summarize this section, the crucial factor limiting the accuracy of the method is not the condition number of $W A$, but the norm of the basis coefficients vector. This can be kept small by rescaling Fourier-Bessel functions and by an informed choice of the fundamental solutions curve. 
8. Conclusions. In this paper we have demonstrated that efficient and highly accurate computation of scattering from a variety of convex and nonconvex polygons can be achieved by using a small number of elements, one for each corner, with the right kind of basis functions on each element. This allows one to tackle problems tens of wavelengths across quite rapidly, even with dense linear algebra. An advantage of the latter is that a dense factorization would allow multiple right-hand sides (e.g., incident wave directions) to be solved at minimal extra cost. Once the coefficients have been solved for, the user may choose where to evaluate the solution at a constant cost per point - this may be slow on large grids, but is trivially parallelizable.

For high-frequency sound-soft scattering of plane waves on convex polygons, the method analyzed in this paper cannot compete with specialized high-frequency boundary element methods (see, e.g., [10]). However, the approach in this paper is much more general. It allows for the computation of convex and nonconvex polygons, soundsoft or sound-hard boundary conditions, and non-plane wave incident fields. Due to its fast exponential convergence, up to medium frequencies ( $\leq 50$ wavelengths in size), it is very competitive.

Our implementation is also much simpler than boundary element or boundary integral methods. At high frequency these usually need a careful treatment of oscillatory integrals with complicated basis functions and weakly oscillatory kernels, or spectral quadrature schemes for (hyper)singular kernels. In addition they require careful meshrefinement or quadrature schemes for corner singularities. None of this complexity is needed in our approach. Moreover, with MPSpack we have created a simple-to-use software tool that allows rapid and flexible implementation of our methods, and more (see Appendix A).

The approach in this paper can easily be generalized to multiple scattering problems. For example, Figure 8.1 shows the solution for sound-soft scattering with incident angle $-\frac{\pi}{3}$ from a disk near a snowflake domain, at wavenumber $k=100$. Setting up and solving the matrix problem takes around 7.5 minutes with an error of $t\left[v_{L S}\right] \approx 4 \cdot 10^{-7}$. Here we used an additional basis of fundamental solutions with charge points lying on a circle inside the disk domain.

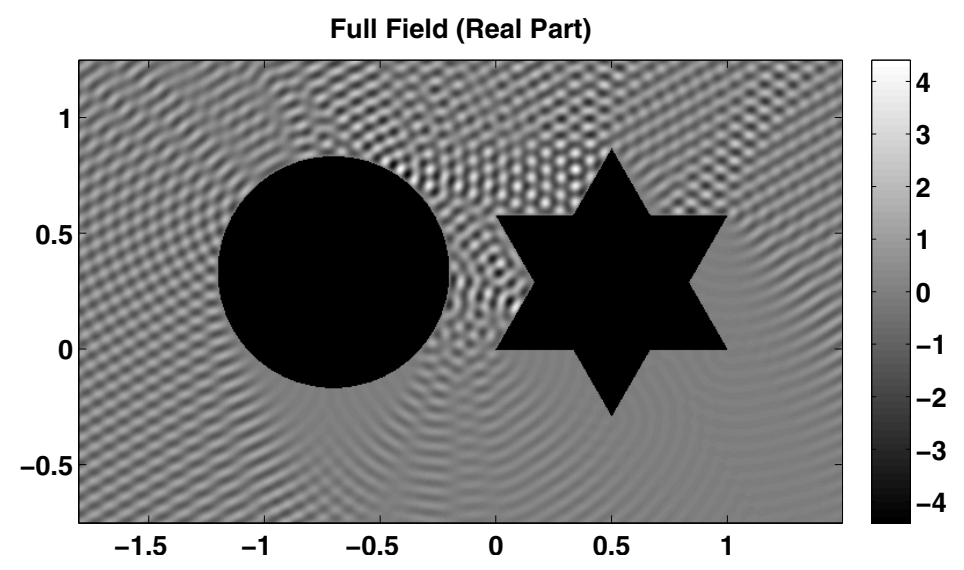

FIG. 8.1. Multiple scattering from a disk and a snowflake domain.

The exponential convergence analyzed in this paper relies on asymptotic series for Helmholtz solutions at corners, and their convergence properties, which are known analytically for sound-soft and sound-hard boundary conditions on straight bound- 
aries. For impedance, transmission, and curved-wall corner problems, the solution is not separable close to a corner, and an open problem is what kind of basis functions to choose here to obtain fast exponential convergence.

Another open problem is optimal mesh (subdomain) generation. The mesh should be chosen in such a way that fast exponential convergence on each element is guaranteed by keeping the relative distance of mesh elements to neighboring singularities large. But at the same time the mesh should not contain too many elements, so that the overall numbers of basis functions and matching points remain small.

Finally, the efficient solution of the least-squares linear algebra problem needs to be further investigated. At the moment we use a dense, backward stable solver, which works well for medium wavenumbers. However, it would be desirable to have iterative solvers for large, structured, ill-conditioned least-squares problems in order to remove the $O\left(k^{3}\right)$ bottleneck at high wavenumbers.

Appendix A. MPSpack example: Scattering from a square. MPSpack is an object-oriented MATLAB toolbox developed by the authors to solve two-dimensional Helmholtz/Laplace PDE problems with particular and fundamental solutions methods. The code can be obtained from http://code.google.com/p/mpspack. The download section of that Web page also includes an extensive tutorial and a technical manual.

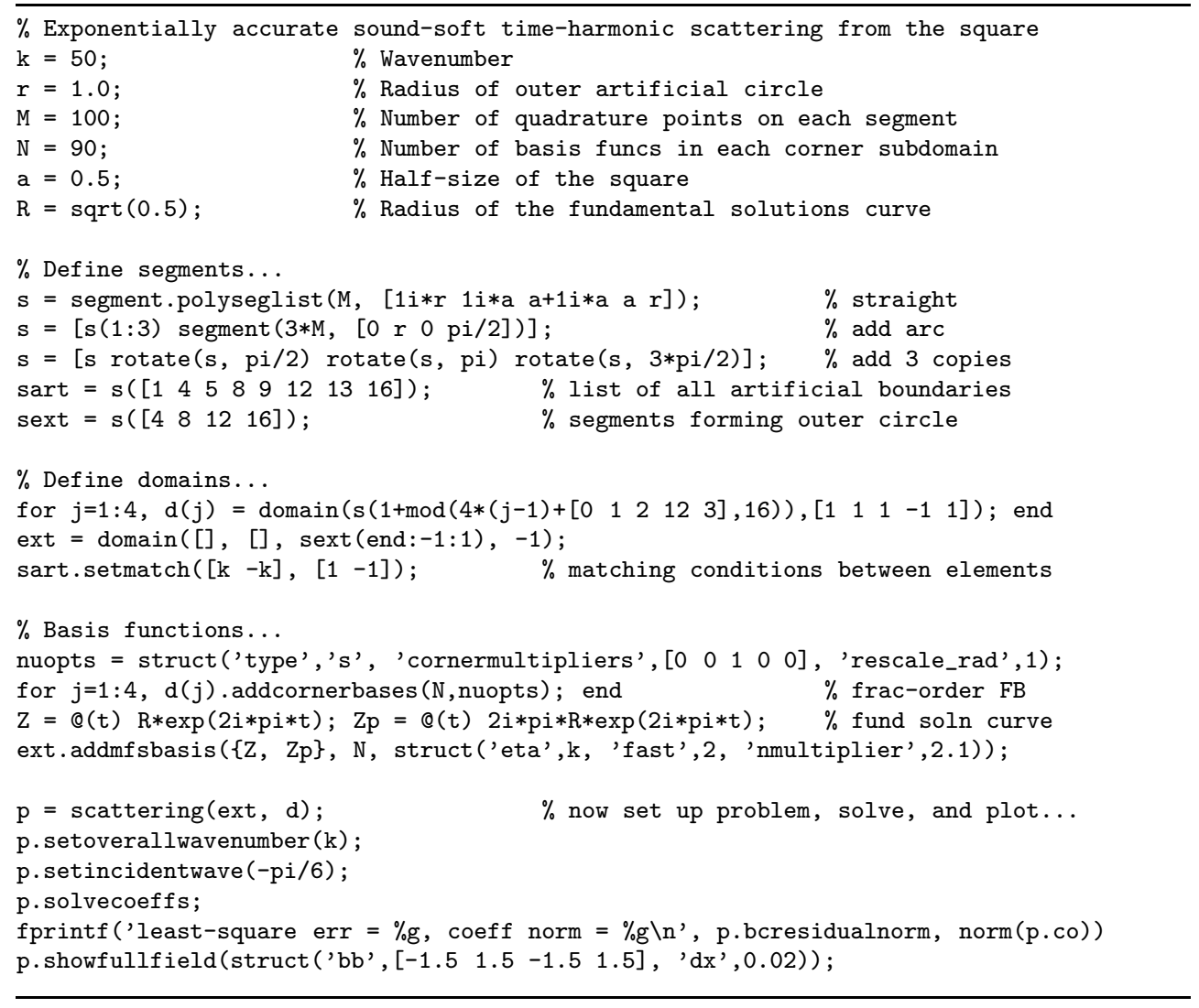

FIG. A.1. MATLAB code for sound-soft scattering from a square using MPSpack toolbox. 
The short, human-readable code in Figure A.1 implements scattering from the square as in section 6.1, giving least-squares error $8 \times 10^{-11}$ in 6 seconds of CPU time, and a further 11 seconds to plot the solution. The code is explained in more detail in the tutorial. Here we will mention only that segments are first created as in Figure 2.1, and then subdomains $E_{1}$ through $E_{4}$ and $\Omega_{e}^{+}$are created. Fractional-order Bessel function bases are added to subdomains with the command addcornerbases, and fundamental solutions are added with addmf sbasis. Finally, a scattering object is created which contains methods to construct the matrix $W A$ from section 3 , solve the least-squares linear system, and plot the solution.

Acknowledgments. Timo Betcke wishes to thank Lehel Banjai (MPI Leipzig), Mahadevan Ganesh (Colorado School of Mines), and Ralf Hiptmair (ETH Zürich) for helpful discussions. Alex Barnett thanks Leslie Greengard (Courant) for helpful discussions. Both authors wish to thank the anonymous referees for helpful comments that improved the presentation of the paper.

\section{REFERENCES}

[1] M. Abramowitz and I. A. Stegun, Handbook of Mathematical Functions with Formulas, Graphs, and Mathematical Tables, 10th ed., Dover, New York, 1964.

[2] E. Anderson, Z. Bai, C. Bischof, S. Blackford, J. Demmel, J. Dongarra, J. Du Croz, A. Greenbaum, S. Hammarling, A. McKenney, and D. Sorensen, LAPACK Users' Guide, 3rd ed., SIAM, Philadelphia, 1999.

[3] I. BabušKa And J. M. Melenk, The partition of unity method, Internat. J. Numer. Methods Engrg., 40 (1997), pp. 727-758.

[4] A. H. BARnett And T. Betcke, Stability and convergence of the method of fundamental solutions for Helmholtz problems on analytic domains, J. Comput. Phys., 227 (2008), pp. 7003-7026.

[5] T. BETcke, A GSVD formulation of a domain decomposition method for planar eigenvalue problems, IMA J. Numer. Anal., 27 (2007), pp. 451-478.

[6] A. Bogomolny, Fundamental solutions method for elliptic boundary value problems, SIAM J. Numer. Anal., 22 (1985), pp. 644-669.

[7] O. P. Bruno, C. A. Geuzaine, J. J. A. Monro, and F. Reitich, Prescribed error tolerances within fixed computational times for scattering problems of arbitrarily high frequency: The convex case, Philos. Trans. R. Soc. Lond. Ser. A Math. Phys. Eng. Sci., 362 (2004), pp. 629645.

[8] O. Cessenat And B. Despres, Application of an ultra weak variational formulation of elliptic PDEs to the two-dimensional Helmholtz problem, SIAM J. Numer. Anal., 35 (1998), pp. 255-299.

[9] S. N. Chandler-Wilde And I. Graham, Boundary integral methods in high frequency scattering, in Highly Oscillatory Problems: Computation, Theory and Applications, B. Engquist, T. Fokas, E. Hairer, and A. Iserles, eds., Cambridge University Press, Cambridge, UK, 2009.

[10] S. N. Chandler-Wilde And S. Langdon, A Galerkin boundary element method for high frequency scattering by convex polygons, SIAM J. Numer. Anal., 45 (2007), pp. 610-640.

[11] D. Colton And R. Kress, Inverse Acoustic and Electromagnetic Scattering Theory, 2nd ed., Appl. Math. Sci. 93, Springer-Verlag, Berlin, 1998.

[12] J. Descloux And M. Tolley, An accurate algorithm for computing the eigenvalues of a polygonal membrane, Comput. Methods Appl. Mech. Engrg., 39 (1983), pp. 37-53.

[13] V. Domínguez, I. G. Graham, and V. P. Smyshlyaev, A hybrid numerical-asymptotic boundary integral method for high-frequency acoustic scattering, Numer. Math., 106 (2007), pp. $471-510$.

[14] S. C. Eisenstat, On the rate of convergence of the Bergman-Vekua method for the numerical solution of elliptic boundary value problems, SIAM J. Numer. Anal., 11 (1974), pp. 654680.

[15] G. Fairweather And A. Karageorghis, The method of fundamental solutions for elliptic boundary value problems, Adv. Comput. Math., 9 (1998), pp. 69-95.

[16] G. Fairweather, A. Karageorghis, and P. A. Martin, The method of fundamental solutions for scattering and radiation problems, Eng. Anal. Bound. Elem., 27 (2003), pp. 759-769. 
[17] P. Gamallo and R. J. Astley, A comparison of two Trefftz-type methods: The ultraweak variational formulation and the least-squares method, for solving shortwave 2-d Helmholtz problems, Internat. J. Numer. Methods Engrg., 71 (2007), pp. 406-432.

[18] C. Geuzaine, O. Bruno, and F. Reitich, On the $O(1)$ solution of multiple-scattering problems, IEEE Trans. Magnetics, 41 (2005), pp. 1488-1491.

[19] P. Henrici, A survey of I. N. Vekua's theory of elliptic partial differential equations with analytic coefficients, Z. Angew. Math. Phys., 8 (1957), pp. 169-203.

[20] P. Henrici, Applied and Computational Complex Analysis. Vol. 3, Pure Appl. Math., John Wiley \& Sons Inc., New York, 1986.

[21] R. Hiptmair, A. Moiola, And I. Perugia, Plane Wave Discontinuous Galerkin Methods for the $2 D$ Helmholtz Equation: Analysis of the p-Version, Tech. Report 2009-20, SAM, ETH Zürich, Switzerland, 2009.

[22] U. Kangro, Convergence of collocation method with delta functions for integral equations of first kind, Integral Equations Operator Theory, 66 (2010), pp. 265-282.

[23] J. R. Kuttler and V. G. Sigillito, Eigenvalues of the Laplacian in two dimensions, SIAM Rev., 26 (1984), pp. 163-193.

[24] P. Monk And D.-Q. WANG, A least-squares method for the Helmholtz equation, Comput. Methods Appl. Mech. Engrg., 175 (1999), pp. 121-136.

[25] F. W. J. Olver, Asymptotics and Special Functions, AKP Classics, A K Peters, Ltd., Wellesley, MA, 1997. Reprint of the 1974 original [Academic Press, New York].

[26] E. Perrey-Debain, Plane wave decomposition in the unit disc: Convergence estimates and computational aspects, J. Comput. Appl. Math., 193 (2006), pp. 140-156.

[27] M. Sтолек, Least-squares Trefftz-type elements for the Helmholtz equation, Internat. J. Numer. Methods Engrg., 41 (1998), pp. 831-849.

[28] I. N. VeKuA, New Methods for Solving Elliptic Equations, OGIZ, Moscow, Leningrad, 1948 (in Russian); North-Holland, Amsterdam, 1967 (in English). 